\title{
On the Asymptotic Distribution of the Scan Statistic FOR EMPIRICAL Distributions
}

\author{
Andrew Ying $\quad$ Wen-Xin Zhou
}

॥

\begin{abstract}
We investigate the asymptotic behavior of several variants of the scan statistic applied to empirical distributions, which can be applied to detect the presence of an anomalous interval with any length. Of particular interest is Studentized scan statistic that is preferable in practice. The main ingredients in the proof are Kolmogorov's theorem, a Poisson approximation, and recent technical results by Kabluchko and Wang [25].
\end{abstract}

\section{Introduction}

The study of the scan statistics dates back1 to Naus [33], who derived the probability that an interval of a certain length contains a certain fraction of independent and identically distributed (iid) samples from the uniform distribution on $[0,1]$. Specifically, let $U_{1}, \ldots, U_{n}$ be iid random variables from $\operatorname{Unif}(0,1)$ with empirical cumulative distribution function (CDF) denoted by $F_{n}$, and let $h$ be the length of the underlying interval of interest. Naus [33] studied the distribution of

$$
\sup _{0 \leq a \leq 1} F_{n}(a+h)-F_{n}(a)
$$

The scan statistics of the uniform empirical distributions can be used to detect elevated signal relative to any continuous null distribution, after an appropriate inverse CDF transformation. Knowing this distribution (11) is essential to calibrating the scan statistic in the context of detecting, in a uniform background, the presence of an interval of a certain length with an unusually high density of points. This is considered today a quintessential detection problem, with applications in the detection of disease clusters [7] and syndromic surveillance [20], among many others [15 18].

In practice, even in the simplest case where only a single anomalous interval may be present, the length of that interval is almost always unknown. In that case, it is natural to consider intervals of various lengths, but standardize the counts, leading to

$$
\sup _{0 \leq a \leq 1} \sup _{h_{-} \leq h \leq h_{+}} \frac{\sqrt{n}\left(F_{n}(a+h)-F_{n}(a)-h\right)}{\sqrt{h(1-h)}} .
$$

This can be seen to approximate the likelihood ratio test [28]. The parameters $h_{-}$and $h_{+}$limit the search to intervals that are neither too short and nor too large. The main goal of this paper is to derive the asymptotic (as $n \rightarrow \infty$ ) distribution of (2) along with its studentized counterpart

$$
\sup _{0 \leq a \leq 1} \sup _{h_{-} \leq F_{n}(a+h)-F_{n}(a) \leq h_{+}} \frac{\sqrt{n}\left(F_{n}(a+h)-F_{n}(a)-h\right)}{\sqrt{\left(F_{n}(a+h)-F_{n}(a)\right)\left(1-F_{n}(a+h)+F_{n}(a)\right)}} .
$$

All authors are with the Department of Mathematics, University of California, San Diego, USA. Contact information is available here and here

${ }^{1}$ Naus himself cites even earlier work in the 1940's by Silberstein [40], Berg [5], and Mack [29]. 
Remark 1. From the four theorems in Section 2, one finds out that relatively small scale $h$ dominates in (2) and (3). There are works that apply scale corrections to the scan [12, 27, 37]. Under the scale corrections, one scale no longer dominates.

\subsection{Related work: point processes}

In one of the most celebrated results in what is now the empirical process literature, Kolmogorov [26] derived the limiting distribution of $\sqrt{n} \sup _{0 \leq a \leq 1}\left(F_{n}(a)-a\right)$. This is the Kolmogorov-Smirnov statistic, and it can be seen as scanning over intervals of the form $[0, a], 0 \leq a \leq 1$.

For similar reasons that motivated the introduction of the normalized scan statistic (2) as an improvement over the unnormalized one (1), Anderson and Darling [2] introduced and studied normalized variants of the Kolmogorov-Smirnov statistic, some of them of the form $\sqrt{n} \sup _{a}\left(F_{n}(a)-\right.$ a) $\sqrt{\psi(a)}$, where $\psi$ is a given weight function. The choice $\psi(a)=[a(1-a)]^{-1}$ is particularly compelling, leading to the statistic

$$
\sup _{0 \leq a \leq 1} \frac{\sqrt{n}\left(F_{n}(a)-a\right)}{\sqrt{a(1-a)}}
$$

Eicker [13] and Jaeschke [21] obtained the limiting distributions of this statistic, its variants of the form

$$
V_{n}=\sup _{\epsilon_{n} \leq a \leq \delta_{n}} \frac{\sqrt{n}\left(F_{n}(a)-a\right)}{\sqrt{a(1-a)}}
$$

and its Studentized counterpart

$$
\hat{V}_{n}=\sup _{\epsilon_{n} \leq a \leq \delta_{n}} \frac{\sqrt{n}\left(F_{n}(a)-a\right)}{\sqrt{F_{n}(a)\left(1-F_{n}(a)\right)}}
$$

for some given $0 \leq \epsilon_{n} \leq \delta_{n} \leq 1$. We note that these statistics can be directly expressed in terms of the order statistics, $U_{(1)} \leq \cdots \leq U_{(n)}$, which when $\varepsilon_{n}=0$ and $\delta_{n}=1$, is as follows

$$
\max _{1 \leq i \leq n} \frac{i-n U_{(i)}}{\sqrt{n U_{(i)}\left(1-U_{(i)}\right)}},
$$

and

$$
\max _{1 \leq i<n} \frac{i-n U_{(i)}}{\sqrt{i\left(1-\frac{i}{n}\right)}},
$$

respectively.

Berk and Jones [6] proposed to directly look at each order statistic individually, combining the resulting tests using Tippett's method, leading to

$$
\min _{1 \leq i \leq n} B\left(U_{(i)} ; i, n-i+1\right),
$$

with $B(\cdot ; a, b)$ denoting the distribution function of the $\operatorname{Beta}(a, b)$ distribution. Moscovich et al. 32] and Gontscharuk and Finner [19] derived the asymptotic distribution of this statistic. Other goodness-of-fit tests include the reversed Berk-Jones statistic [22] and Phi-divergence tests [23], etc.

We note that the two-sided version of the above-mentioned tests have been considered and studied. 


\subsection{Related work: signals}

Closely related to the work above is the setting where, instead of observing a point cloud, one observes a signal. The simplest situation is that of a one-dimensional signal defined on a regular lattice, that is, of the form $X_{1}, \ldots, X_{n}$. The null situation is when these are iid from some underlying distribution on the real line, for example, the standard normal distribution. By writing

$$
R_{n}=\max _{1 \leq i \leq n-k}\left\{S_{i+k}-S_{i}\right\}, \quad k=\lfloor c \log n\rfloor, \quad c>0,
$$

Erdös and Rényi [14] investigated the strong limit of $R_{n} /(\alpha k)$ when $X_{1}$ has a finite moment generating function around a neighborhood of zero. Deheuvels et al. 11] studied limsup and lim inf of $\left(R_{n}-\alpha k\right) / \log k$. When the goal is to detect an interval where the observations are unusually large, and the length of the (discrete) interval is unknown, it becomes of interest to study the following scan statistic

$$
Z_{n}=\max _{1 \leq i<j \leq n} \frac{S_{j}-S_{i}}{\sqrt{j-i}}
$$

where $S_{k}=\sum_{i=1}^{k} X_{i}$.

The study of such statistics dates back to the work of Darling and Erdös [10], who derived the limiting distribution of

$$
\max _{1 \leq j \leq n} \frac{S_{j}}{\sqrt{j}}
$$

which can be seen as scanning intervals of the form $\{1, \ldots, j\}$.

Siegmund and Venkatraman [39] provided the limiting distribution of the statistic (11) under the assumption that the $X_{i}$ 's are iid normal. This study was extended by Mikosch and Račkauskas [31] to the case where the underlying distribution is heavy-tailed, and by Kabluchko and Wang [25] when the underlying distribution has finite moment generating function in a neighborhood of the origin. Kabluchko [24] generalized the result to the multivariate setting where the variables are indexed by a multi-dimensional lattice; see also [27, 37]. Proksch et al. [35] studied more general scanning procedures motivated within the framework of inverse problems.

There is a parallel literature for continuous processes, where one observes instead $X_{t}, t \in[0,1]$ (in dimension 1). See, for example, Aldous [1], Qualls and Watanabe [36] and Chan and Lai [8].

\subsection{Related work: Lipschitz-1/2 modulus of the uniform empirical process}

The results of Mason et al. [30] on the Lipschitz-1/2 modulus of the uniform empirical process, defined by

$$
\sup _{0 \leq a \leq 1-h} \sup _{t \leq h \leq 1} \frac{\sqrt{n}\left|F_{n}(a+h)-F_{n}(a)-h\right|}{\sqrt{h}},
$$

are most closely related to the present results. They proved strong limit theorems for (13) with $t=t_{n} \rightarrow 0$ at various rates. We refer to Shorack and Wellner [38, Chapter 14.2] for a review.

\subsection{Content}

The rest of the paper is organized as follows. We state our main results in Section 2, where we provide asymptotic distributions of some scan statistics and their variants. The proofs are provided in Section 3 , 


\section{Main results}

Recall that $U_{1}, \ldots, U_{n}$ are iid from the uniform distribution on $[0,1]$, and that $U_{(1)} \leq \cdots \leq U_{(n)}$ denote the order statistics. (Whenever needed, we write $U_{(0)} \equiv 0$ and $U_{(n+1)} \equiv 1$.)

\subsection{Studentized scan statistics}

We derive the asymptotics for (3) before (2) for convenience of the proof. As we did earlier, we may rewrite (3) directly in terms of the order statistics, in the form of

$$
M_{n}^{+}(k, l)=\max _{0 \leq i<j \leq n: k \leq j-i<l} M_{i, j}
$$

where

$$
M_{i, j}=\frac{j-i-n\left(U_{(j)}-U_{(i)}\right)}{\sqrt{(j-i)\left(1-\frac{j-i}{n}\right)}} .
$$

We will be particularly interested in the following special case

$$
M_{n}^{+}:=M_{n}^{+}(1, n),
$$

which is the analog of (8). Not surprisingly, the limiting distribution is an extreme value distribution, specifically, a Gumbel distribution. Indeed, we have the following.

Theorem 1. For any $\tau \in \mathbb{R}$,

$$
\lim _{n \rightarrow \infty} \mathbb{P}\left\{M_{n}^{+} \leq \sqrt{2 \log n}-\frac{3 \log \log n}{2 \sqrt{2 \log n}}+\frac{\tau}{\sqrt{2 \log n}}\right\}=\exp (-c \exp (-\tau)),
$$

where $c=\frac{8}{9 \sqrt{\pi}}$.

Similarly, define the opposite one-sided statistics

$$
M_{n}^{-}(k, l)=-\min _{0 \leq i<j \leq n: k \leq j-i \leq l} M_{i, j}
$$

and

$$
M_{n}^{-}:=M_{n}^{-}(1, n)
$$

Finally, define the two-sided statistics

$$
M_{n}(k, l)=\max \left\{M_{n}^{+}(k, l), M_{n}^{-}(k, l)\right\}=\max _{0 \leq i<j \leq n: k \leq j-i<l}\left|M_{i, j}\right|
$$

and

$$
M_{n}:=M_{n}(1, n)=\max \left\{M_{n}^{+}, M_{n}^{-}\right\} .
$$

For these statistics too, the limiting distribution is a Gumbel distribution, but what is surprising here is that these statistics do not behave the same way as $M_{n}^{+}$. In particular, $M_{n}^{-}=(1+$ $\left.o_{P}(1)\right) \log n$, and therefore dominates $M_{n}^{+}$in the large-sample limit, implying that $M_{n}=M_{n}^{-}$with probability tending to 1 . Indeed, we have the following.

Theorem 2. For any $\tau \in \mathbb{R}$,

$$
\lim _{n \rightarrow \infty} \mathbb{P}\left\{M_{n}^{-} \leq \log n+\tau\right\}=\exp (-\exp (1-\tau)) .
$$

Moreover,

$$
\lim _{n \rightarrow \infty} \mathbb{P}\left\{M_{n}=M_{n}^{-}\right\}=1
$$




\subsection{Standardized scan statistics}

We also examine the large-sample behavior of standardized scan statistics (2). Following the same way as rewriting (3) before. Define

$$
\tilde{M}_{n}^{+}(k, l):=\max _{0 \leq i<j \leq n: k \leq j-i \leq l} \tilde{M}_{i, j}
$$

where

$$
\tilde{M}_{i, j}:=\frac{j-i-n\left(U_{(j)}-U_{(i)}\right)}{\sqrt{n\left(U_{(j)}-U_{(i)}\right)\left(1-U_{(j)}+U_{(i)}\right)}} .
$$

Note that

$$
\tilde{M}_{n}^{+}:=\tilde{M}_{n}^{+}(1, n)
$$

is the analog of (17).

The behavior of $\tilde{M}_{n}^{+}$turns out to be very different from that of its studentized analog $M_{n}^{+}$. However, we recover a similar behavior if we appropriately bound the length of the scanning interval from below.

Theorem 3. For any $\tau>0$,

$$
\lim _{n \rightarrow \infty} \mathbb{P}\left\{\tilde{M}_{n}^{+} \leq \sqrt{\frac{n}{\tau}}\right\}=\exp (-\tau)
$$

Moreover, for any $A>0$, defining $k_{n}=\left\lceil A(\log n)^{3}\right\rceil$,

$$
\lim _{n \rightarrow \infty} \mathbb{P}\left\{\tilde{M}_{n}^{+}\left(k_{n}, n\right) \leq \sqrt{2 \log n}-\frac{3 \log \log n}{2 \sqrt{2 \log n}}+\frac{\tau}{\sqrt{2 \log n}}\right\}=\exp \left(-c_{A} \exp (-\tau)\right),
$$

where $c_{A}=\int_{A}^{\infty} \Lambda_{1}(a) d a$ with $\Lambda_{1}(a)=\frac{1}{2 \sqrt{\pi} a^{2}} \exp \left(\frac{\sqrt{2}}{3 \sqrt{a}}\right)$.

Remark 2. Here we choose $k_{n} \propto(\log n)^{3}$ because we want to examine the behavior of $\tilde{M}^{+}(K, L)$, compared to its counterpart $M^{+}(K, L)$ at the most contributed part, which is reflected in the proof of Theorem 1. For readers who are curious about other choices of $k_{n}$, we note that $\tilde{M}_{i, j}$ behaves like subgaussian, or named as "sublogarithmic" in [25]. Roughly speaking, $\tilde{M}_{n}^{+}\left(k_{n}, n\right)$ will likely to take its maximum around the indices $i, j$ with small length, that is, when $j-i$ is close to $k_{n}$.

Define the standardized analog of (19)

$$
\tilde{M}_{n}^{-}(k, l)=-\min _{0 \leq i<j \leq n: k \leq j-i \leq l} \tilde{M}_{i, j}
$$

with

$$
\tilde{M}_{n}^{-}:=\tilde{M}_{n}^{-}(1, n)
$$

as well as the analog of (21)

$$
\tilde{M}_{n}(k, l)=\max \left\{\tilde{M}_{n}^{+}(k, l), \tilde{M}_{n}^{-}(k, l)\right\},
$$

with

$$
\tilde{M}_{n}:=\tilde{M}_{n}(1, n)=\max \left\{\tilde{M}_{n}^{+}, \tilde{M}_{n}^{-}\right\}
$$


Theorem 4. We have

$$
\lim _{n \rightarrow \infty} \mathbb{P}\left(\tilde{M}_{n}=\tilde{M}_{n}^{+}\right)=1
$$

Thus for any $\tau>0$,

$$
\lim _{n \rightarrow \infty} \mathbb{P}\left(\tilde{M}_{n} \leq \sqrt{\frac{n}{\tau}}\right)=\exp (-\tau)
$$

Remark 3. While the behavior of the Studentized statistic $M_{n}^{+}$is driven by the smallest intervals, this is not as much the case for the standardized statistic $\tilde{M}_{n}^{+}$. Indeed, a large value of $M_{n}^{+}$ comes from some $n\left(U_{(j)}-U_{(i)}\right)$ being large compared to $j-i$, however, $n\left(U_{(j)}-U_{(i)}\right)$ being in the denominator defining $\tilde{M}_{n}^{+}$, its impact is lessened.

\section{Proofs of Main Results}

Our proof arguments are based on standard moderate and large deviation results, Kolmogorov's theorem, a Poisson approximation [3], as well as some technical results developed by Kabluchko and Wang [25] in their study of the limiting distribution of the scan statistic in the form of (11).

\subsection{Preliminaries}

Throughout the paper, we assume that $\left\{X_{k}, k \in \mathbb{Z}\right\}$ are iid distributed with the density,

$$
f(x)=\mathbb{1}(x \leq 1) \exp (x-1)
$$

noting that $-X_{1}+1$ follows standard exponential distribution. This distribution has zero mean and unit variance. Define the two-sided partial sums,

$$
S_{k}^{+}=\sum_{i=1}^{k} X_{i}, \quad S_{0}^{+}=0, \quad S_{-k}^{+}=-\sum_{i=1}^{k} X_{-i}, \quad k \in \mathbb{N}
$$

and

$$
S_{k}^{-}:=-S_{k}^{+} .
$$

They will play a central role in what follows. Define the normalized increments

$$
\begin{gathered}
Z_{i, j}^{ \pm}=\frac{S_{j}^{ \pm}-S_{i}^{ \pm}}{\sqrt{j-i}}, \\
Z_{n}^{ \pm}(k, l):=\max _{1 \leq i<j \leq n: k \leq j-i \leq l} Z_{i, j}^{ \pm}, \quad Z_{n}^{ \pm}:=Z_{n}^{ \pm}(1, n) .
\end{gathered}
$$

Let $\varphi^{ \pm}(t)$ be the cumulant generating functions of $\pm X_{1}$ respectively. We have

$$
\begin{aligned}
& \varphi^{+}(t)=t-\log (1+t), \quad \text { if } t \geq 0 . \\
& \varphi^{-}(t)= \begin{cases}-t-\log (1-t), & \text { if } 0 \leq t \leq 1, \\
\infty, & \text { if } t \geq 1,\end{cases}
\end{aligned}
$$

Also, define $I^{+}(s)$ and $I^{-}(s)$ as the respective Legendre-Fenchel transforms (a.k.a., rate functions). We have

$$
I^{+}(s)= \begin{cases}-s-\log (1-s), & \text { if } 0 \leq s \leq 1 \\ \infty, & \text { if } s \geq 1\end{cases}
$$


and

$$
I^{-}(s)=s-\log (1+s)
$$

with respective Taylor expansions at $0($ as $s \rightarrow 0)$

$$
\begin{aligned}
& I^{+}(s)=s^{2} / 2+s^{3} / 3+o\left(s^{3}\right), \\
& I^{-}(s)=s^{2} / 2-s^{3} / 3+o\left(s^{3}\right) .
\end{aligned}
$$

We also prepare several usefull lemmas. The first two lemmas are well-known moderate and large deviations results [4, 9].

Lemma 1. Let $\left(x_{k}\right)$ be a sequence satisfying $x_{k} \rightarrow \infty$ and $x_{k}=o(\sqrt{k})$ as $k \rightarrow \infty$. Then, as $k \rightarrow \infty$,

$$
\mathbb{P}\left(\frac{S_{k}^{ \pm}}{\sqrt{k}} \geq x_{k}\right) \sim \frac{1}{\sqrt{2 \pi} x_{k}} \exp \left\{-k I^{ \pm}\left(\frac{x_{k}}{\sqrt{k}}\right)\right\} .
$$

Lemma 2. For every $k \in \mathbb{N}$ and $x>0$, we have

$$
\mathbb{P}\left(\frac{S_{k}^{ \pm}}{\sqrt{k}} \geq x\right) \leq \exp \left\{-k I^{ \pm}\left(\frac{x}{\sqrt{k}}\right)\right\} .
$$

Moreover, for every $A \leq s_{\infty}$, where $s_{\infty}=\sup \left\{s \in \mathbb{R}: \mathbb{P}\left(X_{1} \leq s\right) \leq 1\right\}$, there is $C_{A}>0$ such that, for all $k \in \mathbb{N}$ and $x \in(0, A \sqrt{k})$,

$$
\mathbb{P}\left(\frac{S_{k}^{ \pm}}{\sqrt{k}} \geq x\right) \leq \frac{C_{A}}{x} \exp \left\{-k I^{ \pm}\left(\frac{x}{\sqrt{k}}\right)\right\}
$$

The following result is obtained from a simple application of Theorem 2.4 in [34], which provides an upper bound of the tail distribution of $\max _{1 \leq k \leq n} S_{k}^{ \pm}$by that of $S_{n}^{ \pm}$.

Lemma 3. We have

$$
\mathbb{P}\left\{\max _{1 \leq k \leq n} S_{k}^{ \pm} \geq x\right\} \leq 2 \mathbb{P}\left\{S_{n}^{ \pm} \geq x-\sqrt{2(n-1)}\right\}
$$

For completeness, we include Lemma 4.4 and 4.5 from [25] below. For integers $r>0$ and $x<y$, define

$$
\mathbb{T}_{r}(x, y):=\{(i, j) \in \mathbb{I}: x-r \leq i \leq x \text { and } y \leq j \leq y+r\} .
$$

Lemma 4. Fix constants $B_{1}, B_{2}>0$. Then for all $x \in \mathbb{Z}, l, r \in \mathbb{N}$ and all $u>0$ such that $B_{1} l>u^{2}$ and $r \leq B_{2} l u^{-2}$, we have

$$
\mathcal{Q}(l, r, u):=\mathbb{P}\left(\max _{i, j \in \mathbb{T}_{r}(x, x+l)} \frac{S_{j}^{+}-S_{i}^{+}}{\sqrt{l}} \geq u\right) \leq \frac{C}{u} \exp \left(-\frac{u^{2}}{2}-\frac{c u^{3}}{\sqrt{l}}\right),
$$

where the constants $c$ and $C$ depend on $B_{1}$ and $B_{2}$ but do not depend on $x, l, r, u$.

Lemma 5. Let $\nu, \nu_{n}, n \in \mathbb{N}$, be measures on $[0, \infty)$ which are finite on compact intervals. Let $G$, $G_{n}, n \in \mathbb{N}$, be measurable functions on $[0, \infty)$ which are uniformly bounded on compact intervals. Assume that

1. $\nu_{n}$ converges to $\nu$ weakly on every interval $[0, t], t \geq 0$;

2. for $\nu$-a.e. $s \geq 0$, we have $\lim _{n \rightarrow \infty} G_{n}\left(s_{n}\right)=G(s)$, for every sequence $s_{n} \rightarrow s$; 
3. $\lim _{T \rightarrow \infty} \int_{T}^{\infty}\left|G_{n}\right| d \nu_{n}=0$ uniformly when $n \geq N$ for some $N \in \mathbb{N}$.

Then, $\lim _{n \rightarrow \infty} \int_{0}^{T} G_{n} d \nu_{n}=\int_{0}^{T} G d \nu$.

We also provide an upper bound of the tail distribution $\max _{i, j \in \mathbb{T}_{r}(x, x+l)}\left(S_{j}^{-}-S_{i}^{-}\right) / \sqrt{l}$ also, which is cruder than its counterpart for $S_{k}^{+}$in Lemma 4 but shall suffice for our purposes.

Lemma 6. For all $x \in \mathbb{Z}, l, r \in \mathbb{N}^{+}$and all $u>40$ such that $l>u^{2} r$ and $r>10 u^{2}$, we have

$$
\mathcal{Q}(l, r, u):=\mathbb{P}\left(\max _{i, j \in \mathbb{T}_{r}(x, x+l)} \frac{S_{j}^{-}-S_{i}^{-}}{\sqrt{l}} \geq u\right) \leq C \exp \left(-\frac{u^{2}}{3}\right),
$$

where the constant $C$ does not depend on $x, l, r, u$.

Proof. Before we proceed into the proof, one fact about $I^{-}(s)$ is

$$
I^{-}(s) \geq \frac{1.01 s^{2}}{3}, \quad 0 \leq s \leq 0.5
$$

which can be easily checked. Define $V_{l, u}:=u^{2}-u S_{l}^{-} / \sqrt{l}, S_{k_{1}}^{(1)-}$ and $S_{k_{2}}^{(2)-}$ to be two partial sums of $-X_{i}$ independent of each other and $S_{l}^{-}$. With translation invariance, we bound $\mathcal{Q}(l, r, u)$ as follows,

$$
\begin{aligned}
\mathcal{Q}(l, r, u) & =\mathbb{P}\left(\max _{i, j \in \mathbb{T}_{r}(0,0+l)} \frac{S_{j}^{-}-S_{i}^{-}}{\sqrt{l}} \geq u\right) \\
& =\mathbb{P}\left(\max _{0 \leq k_{1}, k_{2} \leq r} \frac{S_{k_{1}}^{(1)-}+S_{k_{2}}^{(2)-}}{\sqrt{l}}+\frac{S_{l}^{-}}{\sqrt{l}} \geq u\right) \\
& =\mathbb{P}\left(\max _{0 \leq k_{1}, k_{2} \leq r} \frac{S_{k_{1}}^{(1)-}+S_{k_{2}}^{(2)-}}{\sqrt{l}} \geq \frac{V_{l, u}}{u}\right) \\
& \leq \mathbb{P}\left(\max _{0 \leq k_{1}, k_{2} \leq r} \frac{S_{k_{1}}^{(1)-}+S_{k_{2}}^{(2)-}}{\sqrt{l}} \geq \frac{V_{l, u}}{u}, V_{l, u} \leq u^{2} \sqrt{\frac{r}{l}}\right) \\
& +\mathbb{P}\left(\max _{0 \leq k_{1}, k_{2} \leq r} \frac{S_{k_{1}}^{(1)-}+S_{k_{2}}^{(2)-}}{\sqrt{l}} \geq \frac{V_{l, u}}{u}, V_{l, u}>u^{2} \sqrt{\frac{r}{l}}\right) \\
& \leq \mathbb{P}\left(V_{l, u} \leq u^{2} \sqrt{r / l}\right)+\mathbb{P}\left(\max _{0 \leq k_{1}, k_{2} \leq r} \frac{S_{k_{1}}^{(1)-}+S_{k_{2}}^{(2)-}}{\sqrt{l}}>u \sqrt{\frac{r}{l}}\right)
\end{aligned}
$$

where we bound these two terms individually. By the assumptions on $u, l, r$, we have $u(1-$ $\sqrt{r / l}) / \sqrt{l} \leq 0.5$. Thus with (45) and (51), we have

$$
\mathbb{P}\left(V_{l, u} \leq u^{2} \sqrt{\frac{r}{l}}\right)=\mathbb{P}\left(\frac{S_{l}^{-}}{\sqrt{l}} \geq u-u \sqrt{\frac{r}{l}}\right) \leq \exp \left[-l I^{+}\left\{\frac{u(1-\sqrt{r / l})}{\sqrt{l}}\right\}\right] \leq \exp \left(-\frac{u^{2}}{3}\right) .
$$

Now we switch to the second item, with Lemma 3, (45) and assumption that $r>10 u^{2}, u>40$,

$$
\begin{aligned}
\mathbb{P}\left(\max _{0 \leq k_{1}, k_{2} \leq r} \frac{S_{k_{1}}^{(1)-}+S_{k_{2}}^{(2)-}}{\sqrt{l}} \geq u \sqrt{\frac{r}{l}}\right) & \leq 2 \mathbb{P}\left(\max _{0 \leq k \leq r} \frac{S_{k}^{-}}{\sqrt{r}} \geq \frac{u}{2}\right) \\
& \leq 4 \mathbb{P}\left(\frac{S_{r}^{-}}{\sqrt{r}} \geq \frac{u}{2}-\sqrt{2}\right) \\
& \leq C \exp \left\{-r I^{-}\left(\frac{u-2 \sqrt{2}}{2 \sqrt{r}}\right)\right\} \\
& \leq C \exp \left(-\frac{u^{2}}{3}\right) .
\end{aligned}
$$


Putting the two terms together, we get the stated bound.

We now adjust the Lemma 4 to suit for proving Theorem 3, in which we need to deal with

$$
\tilde{Z}_{i, j}^{+}:=\frac{S_{j}^{+}-S_{i}^{+}}{\sqrt{j-i-\left(S_{j}^{+}-S_{i}^{+}\right)}} .
$$

Define a function

$$
\phi(x)=\frac{x}{\sqrt{1-x}}, \quad x<1,
$$

and thus we have

$$
\frac{\tilde{Z}_{i, j}^{+}}{\sqrt{j-i}}=\phi\left(\frac{Z_{i, j}^{+}}{\sqrt{j-i}}\right) .
$$

Since $\phi(x)$ is strictly increasing on $(-\infty, 1)$ with range $\mathbb{R}$, we write its inverse function as

$$
g^{+}(x):=\frac{1}{2}\left(x \sqrt{x^{2}+4}-x^{2}\right), \quad x \in \mathbb{R},
$$

which is also strictly increasing. Therefore, $\tilde{Z}_{i, j}^{+} \geq u$ if and only if

$$
Z_{i, j}^{+} \geq \sqrt{j-i} \cdot g^{+}\left(\frac{a}{\sqrt{j-i}}\right)
$$

This is an important transformation which enables us to deal with $Z_{i, j}^{+}$instead. We compute the Taylor expansion of $I^{+}\left(g^{+}(s)\right)$ at $s=0$,

$$
I^{+}\left(g^{+}(s)\right)=\frac{s^{2}}{2}-\frac{s^{3}}{6}+O\left(s^{4}\right)
$$

We have

Lemma 7. Fix constants $B_{1}, B_{2}>0$. Then for all $x \in \mathbb{Z}, l, r \in \mathbb{N}$ and all $u>0$ such that $B_{1} l>u^{2}$ and $r<B_{2} l u^{-2}$, we have

$$
\mathcal{Q}(l, r, u):=\mathbb{P}\left(\max _{(i, j) \in \mathbb{T}_{r}(x, x+l)} \tilde{Z}_{i, j}^{+} \geq u\right) \leq \frac{C}{u} \exp \left(-\frac{u^{2}}{2}+\frac{c u^{3}}{\sqrt{l}}\right),
$$

where the constants $c, C>0$ depend on $B_{1}$ and $B_{2}$ but do not depend on $x, l, r, u$.

Proof. By the transformation (57), translation invariance and the fact that $g^{+}(x) / x^{2}$ is strictly decreasing,

$$
\begin{aligned}
\mathcal{Q}(l, r, u) & =\mathbb{P}\left(\max _{(i, j) \in \mathbb{T}_{r}(0, l)} \tilde{Z}_{i, j}^{+} \geq u\right) \\
& =\mathbb{P}\left[\max _{0 \leq k_{1}, k_{2} \leq r}\left\{S_{k_{1}}^{(1)+}+S_{k_{2}}^{(2)+}-\left(l+k_{1}+k_{2}\right) \cdot g^{+}\left(\frac{u}{\sqrt{l+k_{1}+k_{2}}}\right)\right\}+S_{l}^{+} \geq 0\right] \\
& \leq \mathbb{P}\left[\max _{0 \leq k_{1}, k_{2} \leq r}\left\{S_{k_{1}}^{(1)+}+S_{k_{2}}^{(2)+}\right\}-l \cdot g^{+}\left(\frac{u}{\sqrt{l}}\right)+S_{l}^{+} \geq 0\right],
\end{aligned}
$$

where $S_{k_{1}}^{(1)+}, S_{k_{2}}^{(2)+}$ are two partial sums of $X_{i}$ independent of each other and $S_{l}^{+}$. Define

$$
V_{l, u}=u\left(u-\frac{S_{l}^{+}}{\sqrt{l-S_{l}^{+}}}\right) .
$$


Thus

$$
\frac{S_{l}^{+}}{\sqrt{l-S_{l}^{+}}}=\frac{l \cdot S_{l}^{+} / l}{\sqrt{l} \sqrt{1-S_{l}^{+} / l}}=\sqrt{l} \cdot \phi\left(\frac{S_{l}^{+}}{l}\right)=u-\frac{V_{l, u}}{u}
$$

which gives

$$
S_{l}^{+}=l \cdot g^{+}\left(\frac{u-V_{l, u} / u}{\sqrt{l}}\right) .
$$

Therefore,

$$
\begin{aligned}
& \mathcal{Q}(l, r, u) \\
& \leq \mathbb{P}\left[\max _{0 \leq k_{1}, k_{2} \leq r}\left\{S_{k_{1}}^{(1)+}+S_{k_{2}}^{(2)+}\right\}-l \cdot g^{+}\left(\frac{u}{\sqrt{l}}\right)+l \cdot g^{+}\left(\frac{u-V_{l, u} / u}{\sqrt{l}}\right) \geq 0, V_{l, u} \leq 0\right] \\
& +\mathbb{P}\left[\max _{0 \leq k_{1}, k_{2} \leq r}\left\{S_{k_{1}}^{(1)+}+S_{k_{2}}^{(2)+}\right\}-l \cdot g^{+}\left(\frac{u}{\sqrt{l}}\right)+l \cdot g^{+}\left(\frac{u-V_{l, u} / u}{\sqrt{l}}\right) \geq 0, V_{l, u}>0\right] \\
& =\mathbb{P}\left(V_{l, u} \leq 0\right) \\
& +\mathbb{P}\left[\max _{0 \leq k_{1}, k_{2} \leq r}\left\{S_{k_{1}}^{(1)+}+S_{k_{2}}^{(2)+}\right\}-l \cdot g^{+}\left(\frac{u}{\sqrt{l}}\right)+l \cdot g^{+}\left(\frac{u-V_{l, u} / u}{\sqrt{l}}\right) \geq 0, V_{l, u}>0\right] \\
& =F_{l, u}(0)+\int_{0}^{\infty} G_{l, r, u}(s) d F_{l, u}(s),
\end{aligned}
$$

where the last equality is obtained by conditioning on $V_{l, u}=s$, which is independent of $S_{k_{1}}^{(1)+}$, $S_{k_{2}}^{(2)+} \cdot F_{l, u}$ therein is the probability distribution of $V_{l, u}$ and

$$
G_{l, r, u}(s):=\mathbb{P}\left[\max _{0 \leq k_{1}, k_{2} \leq r}\left\{S_{k_{1}}^{(1)+}+S_{k_{2}}^{(2)+}\right\}-l \cdot g^{+}\left(\frac{u}{\sqrt{l}}\right)+l \cdot g^{+}\left(\frac{u-s / u}{\sqrt{l}}\right) \geq 0\right],
$$

which is decreasing. To obtain an upper bound for $\mathcal{Q}(l, r, u)$, first we bound $F_{l, u}(s)$ for $s \in\left[0, \frac{3}{4} u^{2}\right]$ so that $u-s / u \in[u / 4, u]$. Applying (46),

$$
\begin{aligned}
F_{l, u}(s) & =\mathbb{P}\left(\frac{S_{l}^{+}}{\sqrt{l-S_{l}^{+}}} \geq u-\frac{s}{u}\right) \\
& =\mathbb{P}\left\{\frac{S_{l}^{+}}{\sqrt{l}} \geq \sqrt{l} \cdot g^{+}\left(\frac{u-s / u}{\sqrt{l}}\right)\right\} \\
& \leq C\left\{\sqrt{l} \cdot g^{+}\left(\frac{u-s / u}{\sqrt{l}}\right)\right\}^{-1} \exp \left[-l \cdot I^{+}\left\{g^{+}\left(\frac{u-s / u}{\sqrt{l}}\right)\right\}\right] \\
& \leq \frac{C}{u} \exp \left[-l \cdot I^{+}\left\{g^{+}\left(\frac{u-s / u}{\sqrt{l}}\right)\right\}\right],
\end{aligned}
$$

where the last inequality follows from the fact that when $0<x<1$,

$$
x g^{+}\left(\frac{1}{x}\right)>\frac{1}{2} \text {. }
$$

By Taylor expansion of $I^{+}\left(g^{+}(s)\right)$, we have

$$
\begin{aligned}
F_{l, u}(s) & \leq \frac{C}{u} \exp \left\{-\frac{1}{2}\left(u-\frac{s}{u}\right)^{2}+\frac{c}{2 \sqrt{l}}\left(u-\frac{s}{u}\right)^{3}\right\} \\
& \leq \frac{C e^{s}}{u} \exp \left(-\frac{u^{2}}{2}+\frac{c u^{3}}{\sqrt{l}}\right) .
\end{aligned}
$$


It is however easy to see that this inequality continues to hold for $s \geq \frac{3}{4} u^{2}$. Indeed, if $c$ is sufficiently small, then the assumption $B_{1} l>u^{2}$ implies that $c u^{3} / \sqrt{l} \leq u^{2} / 8$. Hence, when $s \geq \frac{3}{4} u^{2}$, the above inequality becomes

$$
F_{l, u}(s) \leq \frac{C}{u} \exp \left(\frac{3 u^{2}}{8}\right)
$$

If $C$ is sufficiently large, the right-hand side of previous inequality is greater than 1 and hence the inequality trivially holds. We bound $G_{l, r, u}(s)$ for $s \geq 0$,

$$
\begin{aligned}
G_{l, r, u}(s) & \leq \mathbb{P}\left\{\max _{0 \leq k_{1}, k_{2}<r} S_{k_{1}}^{(1)+}+S_{k_{2}}^{(2)+}>\frac{s}{2 u} \sqrt{\left(u-\frac{s}{u}\right)^{2}+4 l}+\frac{s^{2}}{2 u^{2}}-s\right\} \\
& \leq 2 \mathbb{P}\left\{\max _{0 \leq k<r} S_{k}^{+}>\frac{s}{4 u} \sqrt{\left(u-\frac{s}{u}\right)^{2}+4 l}-\frac{s}{2}\right\} \\
& \leq 2 \mathbb{P}\left\{\max _{0 \leq k<r} S_{k}^{+}>\frac{s}{2 u} \sqrt{l}-\frac{s}{2}\right\} .
\end{aligned}
$$

Applying the Lemma 3 to the above equation we obtain

$$
\begin{aligned}
G_{l, r, u}(s) & \leq 4 \mathbb{P}\left(S_{r}^{+}>\frac{s}{2 u} \sqrt{l}-\frac{s}{2}-\sqrt{2 r}\right) \\
& \leq 4 \mathbb{P}\left(\frac{S_{r}^{+}}{\sqrt{r}}>\frac{s}{2 u \sqrt{r}} \sqrt{l}-\frac{s}{2 \sqrt{r}}-\sqrt{2}\right) \\
& \leq 4 \exp \left\{-r I^{+}\left(\frac{c s-\sqrt{2}}{\sqrt{r}}\right)\right\} .
\end{aligned}
$$

In the second inequality, we used the assumption $r<B_{2} l u^{-2}$. By noticing the fact that $I^{+}(s) \geq$ $s^{2} / 2$, we have

$$
G_{l, r, u}(s) \leq C e^{-c s^{2}} .
$$

Strictly speaking, this is valid only as long as $c s \geq \sqrt{2}$, however, we can choose the constant $C$ so large that (75) continues to hold in the case $c s<\sqrt{2}$. To obtain (159), by (71), (73)), (175), it is clear that

$$
\begin{aligned}
\mathcal{Q}(l, r, u) & \leq F_{l, u}(0)+\sum_{k=0}^{\infty} G_{l, r, u}(k) F_{l, u}(k+1) \\
& \leq \frac{C}{u}\left(1+\sum_{k=0}^{\infty} e^{-c k^{2}} e^{k}\right) \exp \left(-\frac{u^{2}}{2}+\frac{c u^{3}}{\sqrt{l}}\right) \\
& \leq \frac{C}{u} \exp \left(-\frac{u^{2}}{2}+\frac{c u^{3}}{\sqrt{l}}\right) .
\end{aligned}
$$

\subsection{Proof of Theorem 1 and Theorem 2}

The roadmap of our proof. We know that $\left(U_{(1)}, U_{(2)}, \ldots, U_{(n)}\right)$ has the same distribution as

$$
\left(\frac{Y_{1}}{\sum_{i=1}^{n+1} Y_{i}}, \frac{Y_{1}+Y_{2}}{\sum_{i=1}^{n+1} Y_{i}}, \ldots, \frac{\sum_{i=1}^{n} Y_{i}}{\sum_{i=1}^{n+1} Y_{i}}\right), \text { where } Y_{1}, \ldots, Y_{n+1} \text { are iid exponential. }
$$


In particular, $Y_{i}$ can be set as $1-X_{i}$. We use this fact, together with a comparison of $\sum_{i=1}^{n+1} Y_{i}$ with its mean using a central limit theorem, to deal with the dependency among order statistics above, effectively reducing the problem to partial sums of iid random variables. We then divide the intervals into smaller intervals, which end up contributing the most to the maximum, and larger intervals, whose contribution we show to be negligible. Although $U_{(i)}$ and $Y_{i}$ may be defined on different probability spaces with different probability measure, we may switch between them when there is no confusion. Because we only prove convergence in distribution, from now on, we put $U_{(j)}=\sum_{i=1}^{j} Y_{i} / \sum_{i=1}^{n+1} Y_{i}$ throughout the proof.

\subsubsection{Proof of (17)}

We study the asymptotic behavior of the statistic based on different regions of $j-i$. For $b>0$, define the event

$$
\begin{aligned}
A_{i, j}^{n+}(b) & =\left\{\frac{j-i-n\left(U_{(j)}-U_{(i)}\right)}{\sqrt{(j-i)\left(1-\frac{j-i}{n}\right)}} \leq b\right\} \\
& =\left\{U_{(j)}-U_{(i)} \geq \frac{j-i}{n}-\frac{b}{\sqrt{n}} w_{i, j}^{n}\right\},
\end{aligned}
$$

where

$$
w_{i, j}^{n}:=\sqrt{\frac{j-i}{n}\left(1-\frac{j-i}{n}\right)} .
$$

Under this notation, we have

$$
\left\{M_{n}^{+} \leq b\right\}=\bigcap_{0 \leq i<j \leq n} A_{i, j}^{n+}(b) .
$$

Define

$$
u_{n}(\tau)=\left(1+\frac{-3 \log \log n+2 \tau}{4 \log n}\right) \sqrt{2 \log n}
$$

Throughout the proof, we abbreviate $u_{n}(\tau)$ as $u_{n}$ with $\tau$ fixed. With this choice, we have $u_{n} \sim$ $\sqrt{2 \log n}$.

Step 1: Upper bound For the upper bound, it suffices to focus on the optimal range so that the maximum is achieved. This turns out to be at $j-i \propto(\log n)^{3}$, as discussed below.

Define the events

$$
\Omega_{n}=\left\{\left|S_{n+1}^{+}\right| \leq(\log \log n) \sqrt{n}\right\} .
$$

By the central limit theorem,

$$
\mathbb{P}\left(\Omega_{n}\right) \rightarrow 1 \text { as } n \rightarrow \infty .
$$


When $j-i \leq \frac{n}{\log n \log \log n}$,

$$
\begin{aligned}
& A_{i, j}^{n+}\left(u_{n}\right) \\
& \subseteq \Omega_{n}^{\mathrm{c}} \bigcup\left\{\Omega_{n} \bigcap A_{i, j}^{n+}\left(u_{n}\right)\right\} \\
& =\Omega_{n}^{\mathrm{c}} \bigcup\left(\Omega_{n} \bigcap\left\{\frac{j-i-S_{j}^{+}+S_{i}^{+}}{n+1-S_{n+1}^{+}} \geq \frac{j-i}{n}-\frac{u_{n}}{\sqrt{n}} w_{i, j}^{n}\right\}\right) \\
& \subseteq \Omega_{n}^{\mathrm{c}} \bigcup\left(\Omega_{n} \bigcap\left\{S_{j}^{+}-S_{i}^{+} \leq(j-i) \frac{-1+S_{n+1}^{+}}{n}+\frac{u_{n}}{\sqrt{n}}\left(n+1-S_{n+1}^{+}\right) w_{i, j}^{n}\right\}\right) \\
& \subseteq \Omega_{n}^{\mathrm{c}} \bigcup\left\{S_{j}^{+}-S_{i}^{+} \leq(j-i) \frac{\log \log n}{\sqrt{n}}+\frac{u_{n}}{\sqrt{n}}(n+1+(\log \log n) \sqrt{n}) w_{i, j}^{n}\right\} \\
& =\Omega_{n}^{\mathrm{c}} \bigcup\left\{Z_{i, j}^{+} \leq \frac{(\log \log n)}{\sqrt{n}} \sqrt{j-i}+u_{n} \cdot\left(1+\frac{(\log \log n) \sqrt{n}+1}{n}\right) \sqrt{1-\frac{j-i}{n}}\right\} \\
& \subseteq \Omega_{n}^{\mathrm{c}} \bigcup\left\{Z_{i, j}^{+} \leq \sqrt{\left.\frac{\log \log n}{\log n}+u_{n} \cdot\left(1+\frac{(\log \log n) \sqrt{n}+1}{n}\right)\right\}}\right. \\
& \subseteq \Omega_{n}^{\mathrm{c}} \bigcup\left\{Z_{i, j}^{+} \leq u_{n}(\tau+\varepsilon)\right\},
\end{aligned}
$$

for any fixed $\varepsilon>0$ provided that $n$ is large enough. To deal with the standardized sums $Z_{i, j}^{+}$, we need Theorem 1.1 and Theorem 1.2 in [25]. Because $X_{1} \leq 1$, it belongs to the superlogarithm family defined in [25]. Applying Theorem 1.1 and Theorem 1.2 in [25], we obtain

$$
\lim _{n \rightarrow \infty} \mathbb{P}\left\{Z_{n}^{+} \leq u_{n}\right\}=\exp \left\{-\frac{8}{9 \sqrt{\pi}} e^{-\tau}\right\},
$$

and

$$
\lim _{A \rightarrow \infty} \liminf _{n \rightarrow \infty} \mathbb{P}\left\{Z_{n}^{+}=Z_{n}^{+}\left(A^{-1}(\log n)^{3}, A(\log n)^{3}\right)\right\}=1 .
$$

By (81), (82) and the fact that $(\log n)^{3} \ll \frac{n}{\log n(\log \log n)}$,

$$
\begin{aligned}
& \limsup _{n \rightarrow \infty} \mathbb{P}\left(M_{n}^{+} \leq u_{n}\right) \\
& =\limsup _{n \rightarrow \infty} \mathbb{P}\left\{\bigcap_{0 \leq i<j \leq n} A_{i, j}^{n+}\left(u_{n}\right)\right\} \\
& \leq \limsup _{n \rightarrow \infty} \mathbb{P}\left\{\bigcap_{0 \leq i<j \leq n: j-i \leq \frac{n}{\log n \log \log n}} A_{i, j}^{n+}\left(u_{n}(\tau+\varepsilon)\right)\right\}+\limsup _{n \rightarrow \infty} \mathbb{P}\left(\Omega_{n}^{c}\right) \\
& \leq \limsup _{n \rightarrow \infty} \mathbb{P}\left\{Z_{n}^{+}\left(1, \frac{n}{\log n \log \log n}\right) \leq u_{n}(\tau+\varepsilon)\right\}+\limsup _{n \rightarrow \infty} \mathbb{P}\left(\Omega_{n}^{c}\right) \\
& =\exp \left\{-\frac{8}{9 \sqrt{\pi}} e^{-\tau-\varepsilon}\right\} .
\end{aligned}
$$

As $\varepsilon>0$ is arbitrary we get

$$
\limsup _{n \rightarrow \infty} \mathbb{P}\left(M_{n}^{+} \leq u_{n}\right) \leq \lim _{\varepsilon \rightarrow 0} \exp \left\{-\frac{8}{9 \sqrt{\pi}} e^{-\tau-\varepsilon}\right\}=\exp \left\{-\frac{8}{9 \sqrt{\pi}} e^{-\tau}\right\} \text {. }
$$


Step 2: Lower bound Define

$$
k_{n}=\frac{n}{\log n(\log \log n)}, \quad K_{n}=\frac{n \log \log n}{\log n} .
$$

We establish the lower bound by dividing the range of $j-i$ into five regions:

$$
\begin{array}{ll}
R_{1}=\left[1, u_{n}^{2}\right), & R_{2}=\left[u_{n}^{2}, k_{n}\right), \\
R_{3}=\left[k_{n}, K_{n}\right), & R_{4}=\left[K_{n}, n-K_{n}\right), \\
R_{5}=\left[n-K_{n}, n\right) . &
\end{array}
$$

- For $R_{1}$, note that

$$
\frac{j-i}{n}-\frac{u_{n}}{\sqrt{n}} w_{i, j}^{n} \leq 0,
$$

is equivalent to

$$
j-i \leq \frac{u_{n}^{2}}{1+u_{n}^{2} / n} .
$$

Since $u_{n}^{4} \ll n, i, j$ only take value in integers, it is further equivalent to $j-i \leq u_{n}^{2}$ when $n$ is large enough, which is exactly $R_{1}$. Therefore, when $n$ is large enough,

$$
A_{i, j}^{n+}\left(u_{n}\right)=\Omega,
$$

for any $(i, j)$ satisfying $j-i \in R_{1}$ so that

$$
\bigcap_{0 \leq i<j \leq n: j-i \in R_{1}} A_{i, j}^{n+}\left(u_{n}\right)=\Omega .
$$

- For $R_{2}$, following the same argument that was used to prove the upper bound, it can be shown that

$$
\liminf _{n \rightarrow \infty} \mathbb{P}\left\{\bigcap_{0 \leq i<j \leq n: j-i \in R_{2}} A_{i, j}^{n+}\left(u_{n}\right)\right\} \geq \exp \left\{-\frac{8}{9 \sqrt{\pi}} e^{-\tau}\right\} .
$$

- Turning to $R_{3}$, we shall show that

$$
\mathbb{P}\left(\max _{0 \leq i \leq n-k_{n}} \frac{S_{i+k_{n}}^{+}-S_{i}^{+}}{\sqrt{k_{n}}} \leq \log \log n\right) \rightarrow 1,
$$

and then use this fact to prove that the maximum of $M_{i, j}^{+}$over $R_{3}$ is ignorable. First we bound $\max _{0 \leq i \leq n-k_{n}}\left(S_{i+k_{n}}^{+}-S_{i}^{+}\right)$. Define

$$
q_{n}=\frac{k_{n}}{(\log \log n)^{2}} \ll k_{n},
$$

and introduce a positive sequence $\varepsilon_{n}$ such that $q_{n} \ll \varepsilon_{n} \ll k_{n}$. Consider the following twodimensional grid with mesh size $q_{n}$ :

$$
\mathcal{J}_{n}=\left\{(x, y) \in q_{n} \mathbb{Z}^{2}: x \in\left[-\epsilon_{n}, n+\epsilon_{n}\right], y-x \in\left[0.9 k_{n}-\epsilon_{n}, 1.1 k_{n}+\epsilon_{n}\right]\right\} .
$$

By the union bound,

$$
\mathbb{P}\left\{Z_{n}^{+}\left(0.9 k_{n}, 1.1 k_{n}\right)>\log \log n\right\} \leq \sum_{(x, y) \in \mathcal{J}_{n}} \mathbb{P}\left\{\max _{(i, j) \in \mathbb{T}_{q_{n}}(x, y)} Z_{i, j}^{+} \geq \log \log n\right\} .
$$


Note that the cardinality of $\mathcal{J}_{n}$ satisfies

$$
\left|\mathcal{J}_{n}\right| \sim \frac{(1.1-0.9) n k_{n}}{\left(q_{n}\right)^{2}}=0.2(\log \log n)^{5} \log n .
$$

By the translation invariance property of $\mathbb{T}_{q_{n}}(x, y)$ and Lemma 4, taking $l=y-x, r=q_{n}$ and $u=\log \log n$ for large enough $n$ (and thus satisfying the conditions in Lemma 4) temporarily, we have

$$
\mathbb{P}\left\{Z_{n}^{+}\left(0.9 k_{n}, 1.1 k_{n}\right) \geq \log \log n\right\} \leq C\left|\mathcal{J}_{n}\right| \exp \left\{-\frac{(\log \log n)^{2}}{2}\right\} \rightarrow 0,
$$

where $C>0$ is a constant. Since

$$
\max _{0 \leq i \leq n-k_{n}} \frac{S_{i+k_{n}}^{+}-S_{i}^{+}}{\sqrt{k_{n}}} \leq Z_{n}^{+}\left(0.9 k_{n}, 1.1 k_{n}\right),
$$

it follows that

$$
\limsup _{n \rightarrow \infty} \mathbb{P}\left(\max _{0 \leq i \leq n-k_{n}} \frac{S_{i+k_{n}}^{+}-S_{i}^{+}}{\sqrt{k_{n}}} \geq \log \log n\right)=0 .
$$

We may now prove the ignorability of maximum of $M_{i, j}^{+}$when taking values on $R_{3}$. Define

$$
\Omega_{1 n}:=\left\{\max _{0 \leq i \leq n-k_{n}} \frac{S_{i+k_{n}}^{+}-S_{i}^{+}}{\sqrt{k_{n}}} \leq \log \log n\right\} .
$$

By (97), $\mathbb{P}\left(\Omega_{1 n}\right) \rightarrow 1$ as $n \rightarrow \infty$. For $j-i \in R_{3}$,

$$
\begin{aligned}
& A_{i, j}^{n+}\left(u_{n}\right) \\
& \supseteq \Omega_{n} \bigcap \Omega_{1 n} \bigcap\left\{S_{j}^{+}-S_{i}^{+} \leq(j-i) \frac{S_{n+1}^{+}-1}{n}+\frac{u_{n}}{\sqrt{n}}\left(n+1-S_{n+1}\right) w_{i, j}^{n}\right\} \\
& =\Omega_{n} \bigcap \Omega_{1 n} \bigcap\left\{S_{j}^{+}-S_{i+k_{n}}^{+} \leq(j-i) \frac{S_{n+1}^{+}-1}{n}-S_{i+k_{n}}^{+}+S_{i}^{+}+\frac{u_{n}}{\sqrt{n}}\left(n+1-S_{n+1}^{+}\right) w_{i, j}^{n}\right\} \\
& \supseteq \Omega_{n} \bigcap \Omega_{1 n} \bigcap\left\{S_{j}^{+}-S_{i+k_{n}}^{+} \leq-(j-i) \frac{\log \log n}{\sqrt{n}}-\sqrt{k_{n}} \log \log n+\frac{u_{n}}{\sqrt{n}}(n+1-\log \log n \sqrt{n}) w_{i, j}^{n}\right\} \\
& \supseteq \Omega_{n} \bigcap \Omega_{1 n} \bigcap\left\{\frac{S_{j}^{+}-S_{i+k_{n}}^{+}}{\sqrt{j-i-k_{n}}} \leq \sqrt{\frac{j-i}{j-i-k_{n}}}\left[u_{n} \cdot\left(1-\frac{\log \log n}{\sqrt{n}}\right)-\sqrt{\frac{(\log \log n)^{3}}{\log n}}-\log \log n\right]\right\} \\
& \supseteq \Omega_{n} \bigcap \Omega_{1 n} \bigcap\left\{\frac{S_{j}^{+}-S_{i+k_{n}}^{+}}{\sqrt{j-i-k_{n}}} \leq \sqrt{1+\frac{k_{n}}{K_{n}}}\left[u_{n} \cdot\left(1-\frac{\log \log n}{\sqrt{n}}\right)-\sqrt{\left.\left.\frac{(\log \log n)^{3}}{\log n}-\log \log n\right]\right\}}\right.\right. \\
& \supseteq \Omega_{n} \bigcap \Omega_{1 n} \bigcap\left\{\frac{S_{j}^{+}-S_{i+k_{n}}^{+}}{\sqrt{j-i-k_{n}}} \leq u_{n}(\log \log n)\right\},
\end{aligned}
$$

where the last line follows by noting that $k_{n} / K_{n}=1 /(\log \log n)^{2}$. Thus

$$
\begin{aligned}
& \bigcap_{0 \leq i<j \leq n: k_{n}+1 \leq j-i \leq K_{n}} A_{i, j}^{n+}\left(u_{n}\right) \\
& \supset \Omega_{n} \bigcap \Omega_{1 n} \bigcap\left\{\max _{0 \leq i<j \leq n: k_{n}+1 \leq j-i \leq K_{n}} \frac{S_{j}^{+}-S_{i+k_{n}}^{+}}{\sqrt{j-i-k_{n}}} \leq u_{n}(\log \log n)\right\} \\
& \supset \Omega_{n} \bigcap \Omega_{1 n} \bigcap\left\{\max _{0 \leq i<j \leq n: j-i \leq K_{n}} \frac{S_{j}^{+}-S_{i}^{+}}{\sqrt{j-i}} \leq u_{n}(\log \log n)\right\},
\end{aligned}
$$


and recall that $u_{n}(\cdot)$ is a function. Since $(\log n)^{3} \ll K_{n}$, (82) and (83) together imply that

$$
\begin{aligned}
& \liminf _{n \rightarrow \infty} \mathbb{P}\left\{M_{n}^{+}\left(k_{n}+1, K_{n}\right) \leq u_{n}(\tau)\right\} \\
& \geq \liminf _{n \rightarrow \infty} \mathbb{P}\left[\Omega_{n} \bigcap \Omega_{1 n} \bigcap\left\{Z_{n}^{+}\left(1, K_{n}\right) \leq u_{n}(\log \log n)\right\}\right] \\
& \geq \liminf _{n \rightarrow \infty} \mathbb{P}\left[\Omega_{n} \bigcap \Omega_{1 n} \bigcap\left\{Z_{n}^{+}\left(1, K_{n}\right) \leq u_{n}\left(\tau^{\prime}\right)\right\}\right]=\exp \left(-\frac{8}{9 \sqrt{\pi}} e^{-\tau^{\prime}}\right),
\end{aligned}
$$

for any $\tau, \tau^{\prime}$. We now take $\tau^{\prime} \rightarrow \infty$, yielding

$$
\liminf _{n \rightarrow \infty} \mathbb{P}\left\{M_{n}^{+}\left(k_{n}+1, K_{n}\right) \leq u_{n}(\tau)\right\}=\liminf _{\tau^{\prime} \rightarrow \infty} \exp \left(-\frac{8}{9 \sqrt{\pi}} e^{-\tau^{\prime}}\right)=1 .
$$

- Next we apply the Kolmogorov's Theorem to deal with $R_{4}$. Define the centered order statistics

$$
\bar{U}_{(i)}=U_{(i)}-\frac{i}{n+1}
$$

Note that when $n$ is large enough,

$$
\begin{aligned}
A_{i, j}^{n+}\left(u_{n}\right) & =\left\{\bar{U}_{(j)}-\bar{U}_{(i)} \geq \frac{j-i}{n(n+1)}-\frac{u_{n}}{\sqrt{n}} w_{i, j}^{n}\right\} \\
& =\left\{\sqrt{n}\left(\bar{U}_{(j)}-\bar{U}_{(i)}\right) \geq \frac{j-i}{\sqrt{n}(n+1)}-u_{n} w_{i, j}^{n}\right\} \\
& \supseteq\left\{\sqrt{n}\left(\bar{U}_{(j)}-\bar{U}_{(i)}\right) \geq-0.9 u_{n} w_{i, j}^{n}\right\} \\
& \supseteq\left\{0.9 u_{n} w_{i, j}^{n} \geq \sqrt{n}\left(\bar{U}_{(j)}-\bar{U}_{(i)}\right) \geq-0.9 u_{n} w_{i, j}^{n}\right\} \\
& \supseteq\left\{2 \sqrt{n} \max \left\{\left|\bar{U}_{(i)}\right|,\left|\bar{U}_{(j)}\right|\right\} \leq 0.9 u_{n} w_{i, j}^{n}\right\} .
\end{aligned}
$$

For $(i, j)$ such that $j-i \in R_{4}, w_{i, j}^{n}$ is minimized at either $j-i=\frac{n \log \log n}{\log n}$ or $n-\frac{n \log \log n}{\log n}$. Consequently,

$$
\begin{aligned}
\bigcap_{0 \leq i<j \leq n: j-i \in R_{4}} A_{i, j}^{n}\left(u_{n}\right) & \supseteq\left\{\sqrt{n} \max _{1 \leq i \leq n}\left\{\left|\bar{U}_{(i)}\right|\right\} \leq \frac{0.9 u_{n}}{2} \min _{0 \leq i<j \leq n: j-i \in R_{4}} w_{i, j}^{n}\right\} \\
& =\left\{\sqrt{n} \max _{1 \leq i \leq n}\left\{\left|\bar{U}_{(i)}\right|\right\} \leq \frac{0.9 u_{n}}{2} \sqrt{\frac{\log \log n}{\log n}\left(1-\frac{\log \log n}{\log n}\right)}\right\} .
\end{aligned}
$$

The Kolmogorov's Theorem states that for any $y \geq 0$,

$$
\lim _{n \rightarrow \infty} \mathbb{P}\left(\sqrt{n} \max _{1 \leq i \leq n}\left|\bar{U}_{(i)}\right| \leq y\right)=K(y):=1-2 e^{-2 y^{2}}+2 e^{-8 y^{2}}-\cdots .
$$

In particular, $\left(\sqrt{n} \max _{1 \leq i \leq n}\left|\bar{U}_{(i)}\right|\right)$ is tight. Therefore, by the fact that

$$
\frac{0.9 u_{n}}{2} \sqrt{\frac{\log \log n}{\log n}\left(1-\frac{\log \log n}{\log n}\right)} \asymp \sqrt{\log \log n} \rightarrow \infty,
$$


we obtain

$$
\lim _{n \rightarrow \infty} \mathbb{P}\left\{\bigcap_{0 \leq i<j \leq n: j-i \in R_{4}} A_{i, j}^{n+}\left(u_{n}\right)\right\}=1 .
$$

- For $R_{5}$, define $j^{\prime}=n-j$ and $U_{\left(j^{\prime}+1\right)}^{\prime}=1-U_{\left(n+1-j^{\prime}-1\right)}=1-U_{(j)}$. A simple change of indices gives

$$
\begin{aligned}
M_{n}^{+}\left(n-K_{n}, n\right) & =\max _{\substack{0 \leq i<j \leq n \\
n-K_{n} \leq j-i<n}} \frac{j-i-n\left(U_{(j)}-U_{(i)}\right)}{\sqrt{(j-i)\left(1-\frac{j-i}{n}\right)}} \\
& \leq \max _{\substack{i, j^{\prime} \geq 0 \\
i+j^{\prime}<K_{n}}} \frac{n U_{\left(j^{\prime}+1\right)}^{\prime}-\left(j^{\prime}+1\right)+n U_{(i)}-i}{\sqrt{\left(i+j^{\prime}\right)\left(1-\frac{i+j^{\prime}}{n}\right)}} \\
& \leq 1.01 \max _{\substack{i, j \geq 0 \\
1 \leq i+j<K_{n}}} \frac{n U_{(j)}^{\prime}-j+n U_{(i)}-i}{\sqrt{i+j}}+1.01
\end{aligned}
$$

where the last inequality holds when $n$ is large enough since $K_{n} \ll n$. Now, by the above statements, to prove

$$
\limsup _{n \rightarrow \infty} \mathbb{P}\left(M_{n}^{+}\left(n-K_{n}, n\right) \geq u_{n}\right)=0
$$

it suffices to prove

$$
\limsup _{n \rightarrow \infty} \mathbb{P}\left(\max _{\substack{i, j \geq 0 \\ 1 \leq i+j<K_{n}}} \frac{n U_{(i)}-i+n U_{(j)}^{\prime}-j}{\sqrt{i+j}} \geq \sqrt{1.9 \log n}\right)=0 .
$$

Assuming $0 / 0=0$, observe that

$$
\begin{aligned}
& \mathbb{P}\left(\max _{\substack{i, j \geq 0 \\
1 \leq i+j<K_{n}}} \frac{n U_{(i)}-i+n U_{(j)}^{\prime}-j}{\sqrt{i+j}} \geq \sqrt{1.9 \log n}\right) \\
& =\mathbb{P}\left\{\max _{\substack{i, j \geq 0 \\
1 \leq i+j \leq K_{n}}}\left(\frac{n U_{(i)}-i}{\sqrt{i+j}}+\frac{n U_{(j)}^{\prime}-j}{\sqrt{i+j}}\right) \geq \sqrt{1.9 \log n}\right\} \\
& \leq \mathbb{P}\left\{\max _{\substack{i, j \geq 0 \\
1 \leq i+j \leq K_{n}}}\left(\frac{n U_{(i)}-i}{\sqrt{i}}+\frac{n U_{(j)}^{\prime}-j}{\sqrt{j}}\right) \geq \sqrt{1.9 \log n}\right\} \\
& \leq \mathbb{P}\left(\max _{0 \leq i \leq n} \frac{n U_{(i)}-i}{\sqrt{i}}+\max _{0 \leq j \leq n} \frac{n U_{(j)}^{\prime}-j}{\sqrt{j}} \geq \sqrt{1.9 \log n}\right) \\
& \leq 2 \mathbb{P}\left(\max _{0 \leq i \leq n} \frac{n U_{(i)}-i}{\sqrt{i}} \geq \frac{\sqrt{1.9 \log n}}{2}\right) \\
& \leq 2 \mathbb{P}\left(\max _{0 \leq i \leq n} \frac{n U_{(i)}-i}{\sqrt{i(1-i / n})} \geq \frac{\sqrt{1.9 \log n}}{2}\right) .
\end{aligned}
$$

However, Eicker [13] showed that

$$
\max _{0 \leq i \leq n} \frac{n U_{(i)}-i}{\sqrt{i(1-i / n)}} \sim \sqrt{2 \log \log n}
$$


which finishes the proof for $R_{5}$.

- Now combining all the results gives the lower bound, which, together with the upper bound, establishes the proof of Theorem 1.

\subsubsection{Proof of (22)}

In what follows, we let

$$
u_{n}=u_{n}(\tau):=\log n+\tau,
$$

with $\tau$ fixed. Define

$$
A_{i, j}^{n-}\left(u_{n}\right)=\left\{\frac{n\left(U_{(j)}-U_{(i)}\right)-(j-i)}{\sqrt{(j-i)\left(1-\frac{j-i}{n}\right)}} \leq u_{n}\right\}=\left\{U_{(j)}-U_{(i)} \leq \frac{j-i}{n}+\frac{u_{n}}{\sqrt{n}} w_{i, j}^{n}\right\}
$$

where $w_{i, j}^{n}$ is defined in (77), and note that

$$
\left\{M_{n}^{-} \leq u_{n}\right\}=\bigcap_{0 \leq i<j \leq n} A_{i, j}^{n-}\left(u_{n}\right)
$$

Step 1: Upper bound For the upper bound, again, we only consider a particular order of magnitude for the length, the one that contributes the most to the maximum. When $j-i \leq \frac{n \log \log n}{(\log n)^{2}}$,

$$
\begin{aligned}
A_{i, j}^{n-}\left(u_{n}\right) & \subset \Omega_{n}^{\mathrm{c}} \bigcup\left\{\Omega_{n} \bigcap A_{i, j}^{n-}\left(u_{n}\right)\right\} \\
& \subset \Omega_{n}^{\mathrm{c}} \bigcup\left\{\frac{S_{j}^{-}-S_{i}^{-}}{\sqrt{j-i}} \leq(\log \log n) \sqrt{\frac{j-i}{n}}+u_{n} \cdot\left(1+\frac{\log \log n}{\sqrt{n}}\right)\right\} \\
& \subset \Omega_{n}^{\mathrm{c}} \bigcup\left\{\frac{S_{j}^{-}-S_{i}^{-}}{\sqrt{j-i}} \leq u_{n}(\tau+\varepsilon)\right\},
\end{aligned}
$$

for any $\varepsilon>0$, where $\Omega_{n}$ is given in (80). By (81), it suffices to consider the second event on the RHS. Applying Theorem 1.7 in [25], the limiting distribution of $Z_{n}^{-}$is the same as that of $\max _{1 \leq i \leq n}\left(-X_{i}\right)$. By the independence of $\left\{X_{i}\right\}$, we obtain

$$
\lim _{n \rightarrow \infty} \mathbb{P}\left(Z_{n}^{-} \leq u_{n}\right)=\lim _{n \rightarrow \infty} \mathbb{P}\left\{\max _{1 \leq i \leq n}\left(-X_{i}\right) \leq u_{n}\right\}=\exp \{-\exp (1-\tau)\} .
$$

Therefore, taking $\varepsilon \rightarrow 0$,

$$
\begin{aligned}
\limsup _{n \rightarrow \infty} \mathbb{P}\left(M_{n}^{-} \leq u_{n}\right) & =\limsup _{n \rightarrow \infty} \mathbb{P}\left\{\bigcap_{0 \leq i<j \leq n} A_{i, j}^{n-}\left(u_{n}\right)\right\} \\
& \leq \limsup _{n \rightarrow \infty} \mathbb{P}\left\{\bigcap_{0 \leq i<j \leq n: j-i \leq \frac{n \log \log n}{(\log n)^{2}}} A_{i, j}^{n-}\left(u_{n}\right)\right\}+\mathbb{P}\left(\Omega_{n}^{\mathrm{c}}\right) \\
& \leq \limsup _{\varepsilon \rightarrow 0} \exp \{-\exp (1-\tau-\varepsilon)\} \\
& =\exp \{-\exp (1-\tau)\} .
\end{aligned}
$$


Step 2: Lower bound As in the proof of (1), we divide the range of $j-i$ into several subintervals. Similar to the upper bound case,

$$
\lim _{n \rightarrow \infty} \mathbb{P}\left\{M_{n}^{-}\left(1, \frac{n \log \log n}{(\log n)^{2}}\right) \leq u_{n}\right\}=\exp \{-\exp (1-\tau)\} .
$$

With the same argument that was used to prove (103), we obtain

$$
\lim _{n \rightarrow \infty} \mathbb{P}\left\{\bigcap_{0 \leq i<j \leq n: \frac{n \log \log n}{(\log n)^{2}} \leq j-i \leq n-\frac{n \log \log n}{(\log n)^{2}}} A_{i, j}^{n-}\left(u_{n}\right)\right\}=1 .
$$

The case where $j-i \geq n-\frac{n \log \log n}{(\log n)^{2}}$ can be treated similarly to proving the region $R_{5}$ in the proof of Theorem 1, even easier since now $u_{n} \sim \log n$ (and details are omitted).

\subsubsection{Proof of $(\underline{23})$}

This follows directly from (17), where we learn that $M_{n}^{+} \asymp_{P} \sqrt{\log n}$, and (22), which states that $M_{n}^{-} \asymp_{P} \log n$ (here $A_{n} \asymp_{P} B_{n}$ means $A_{n}=O\left(B_{n}\right)$ and $B_{n}=O\left(A_{n}\right)$ ). Combining them implies that $M_{n}^{-} \gg_{P} M_{n}^{+}$, and therefore $M_{n}=\max \left(M_{n}^{-}, M_{n}^{+}\right)=M_{n}^{-}$with probability tending to 1 as $n$ increases.

\subsection{Proof of Theorem 3}

\subsubsection{Proof of $(27)$}

We first derive the asymptotic distribution of

$$
\tilde{M}_{n}^{+}(1,2)=\max _{0 \leq i \leq n-1} \frac{1-n\left(U_{(i+1)}-U_{(i)}\right)}{\sqrt{n\left(U_{(i+1)}-U_{(i)}\right)\left(1-U_{(i+1)}+U_{(i)}\right)}},
$$

which is exactly the same as that of (27) and then show that $\tilde{M}_{n}^{+}(2, n) \ll_{P} \sqrt{n}$. These together imply (27). To get the asymptotic distribution of $\tilde{M}_{n}^{+}(1,2)$, note that

$$
\begin{aligned}
\tilde{M}_{n}^{+}(1,2) \leq & \max _{0 \leq i \leq n-1} \frac{1}{\sqrt{n\left(U_{(i+1)}-U_{(i)}\right)\left[1-\left(U_{(i+1)}-U_{(i)}\right)\right.}} \\
& \text { and } \tilde{M}_{n}^{+}(1,2) \geq \max _{0 \leq i \leq n-1} \frac{1-n\left(U_{(i+1)}-U_{(i)}\right)}{\sqrt{n\left(U_{(i+1)}-U_{(i)}\right)}},
\end{aligned}
$$

where both upper and lower bounds are functions of

$$
T:=\min _{0 \leq i \leq n-1}\left(U_{(i+1)}-U_{(i)}\right) .
$$

Therefore it suffices to work on $T$ instead. It is easy to see that $T \leq 1 / n$. By symmetry,

$$
\mathbb{P}(T \geq t)=n ! \mathbb{P}\left(T \geq t, U_{1} \leq U_{2} \leq \cdots \leq U_{n}\right)
$$

Define the subset

$$
A_{t}=\left\{\left(u_{1}, \ldots, u_{n}\right) \in[0,1]^{n}: u_{i}+t \leq u_{i+1}, i=0,1, \ldots, n-1\right\},
$$


where $u_{0}=0$. Then,

$$
\left\{\left(U_{1}, \cdots, U_{n}\right) \in A_{t}\right\}=\left\{T \geq t, U_{1} \leq U_{2} \leq \cdots \leq U_{n}\right\},
$$

and hence

$$
\mathbb{P}\left(T \geq t, U_{1} \leq U_{2} \leq \cdots \leq U_{n}\right)=\lambda_{n}\left(A_{t}\right),
$$

where $\lambda_{n}$ is the Lebesgue measure on $\mathbb{R}^{n}$. Define a mapping

$$
h: \quad A_{t} \longrightarrow Q \subset[0,1-n t]^{n}, \quad h\left(u_{1}, u_{2}, \cdots, u_{n}\right)=\left(u_{1}-t, u_{2}-2 t, u_{n}-n t\right),
$$

where

$$
Q:=\left\{\left(y_{1}, \ldots, y_{n}\right): y_{i} \leq y_{i+1}, \forall 1 \leq i \leq n-1\right\} \cap[0,1-n t]^{n} .
$$

It is easy to verify that $h$ is a volume-preserving bijection. Hence

$$
\mathbb{P}\left(T \geq t, U_{1} \leq U_{2} \leq \cdots \leq U_{n}\right)=\lambda_{n}\left(A_{t}\right)=\lambda_{n}(Q)=\frac{(1-n t)^{n}}{n !}
$$

Therefore, we have

$$
\mathbb{P}(T \geq t)=\frac{n !(1-n t)^{n}}{n !}=(1-n t)^{n},
$$

for $0 \leq t \leq 1 / n$. For any $0 \leq t \leq 1 / n$,

$$
\mathbb{P}\left\{\min _{0 \leq i \leq n-1}\left(U_{(i+1)}-U_{(i)}\right) \geq t\right\}=(1-n t)^{n},
$$

which implies

$$
\lim _{n \rightarrow \infty} \mathbb{P}\left\{\min _{0 \leq i \leq n-1}\left(U_{(i+1)}-U_{(i)}\right) \geq \frac{\tau}{n^{2}}\right\}=\exp (-\tau) .
$$

This, together with (113) and (114), implies

$$
\lim _{n \rightarrow \infty} \mathbb{P}\left(\tilde{M}_{n}^{+}(1,1) \leq \sqrt{\frac{n}{\tau}}\right)=\exp (-\tau)
$$

It remains to show that $\tilde{M}_{n}^{+}(2, n) \ll_{P} \sqrt{n}$. We will divide it into $\tilde{M}_{n}^{+}\left(2,(\log n)^{2}\right), \tilde{M}_{n}^{+}\left((\log n)^{2}, n-\right.$ $\left.(\log n)^{2}\right)$ and $\tilde{M}_{n}^{+}\left(n-(\log n)^{2}, n\right)$. When $2 \leq j-i \leq(\log n)^{2}$, note that

$$
\begin{aligned}
1-\left(U_{(j)}-U_{(i)}\right) & =1-\frac{j-i}{n+1}-\left(\bar{U}_{(j)}-\bar{U}_{(i)}\right) \\
& \geq 1-\frac{(\log n)^{2}}{n+1}-2 \max _{1 \leq i \leq n}\left|\bar{U}_{(i)}\right| \\
& =1+O_{P}(1 / \sqrt{n}) \\
& \geq 0.5,
\end{aligned}
$$

where the last inequality holds on a sequence of events with probability tending to one, by Kolmogorov's Theorem mentioned in the proof of Theorem 1 when $n$ is large enough. Meanwhile,

$$
\begin{aligned}
\frac{j-i-n\left(U_{(j)}-U_{(i)}\right)}{\sqrt{n\left(U_{(j)}-U_{(i)}\right)}} & =\frac{j-i-\frac{n}{n+1-S_{n+1}^{+}}\left(j-i-S_{j}^{+}+S_{i}^{+}\right)}{\sqrt{n \frac{n}{n+1-S_{n+1}^{+}}\left(j-i-S_{j}^{+}+S_{i}^{+}\right)}} \\
& =\left(1+O_{P}(1 / \sqrt{n})\right) \tilde{Z}_{i, j}+O_{P}(1 / \sqrt{n}) \\
& \leq 1.01 \tilde{Z}_{i, j}+0.01,
\end{aligned}
$$


on the sequence of events $\Omega_{n}$ defined in (81). With these results, the union bound, (45) and the fact that $I^{+}(s)=-s-\log (1-s)$ on $[0,1)$, for any $\varepsilon>0$,

$$
\begin{aligned}
& \mathbb{P}\left(\tilde{M}_{n}^{+}\left(2,(\log n)^{2}\right) \geq \varepsilon \sqrt{n}\right) \\
& \leq \mathbb{P}\left(\tilde{Z}_{n}^{+}\left(2,(\log n)^{2}\right) \geq 0.9 \varepsilon \sqrt{n}\right)+\mathbb{P}\left(\Omega_{n}^{c}\right) \\
& \leq \sum_{0 \leq i<j \leq n: 2 \leq j-i \leq(\log n)^{2}} \mathbb{P}\left(\tilde{Z}_{i, j}^{+} \geq 0.9 \varepsilon \sqrt{n}\right)+\mathbb{P}\left(\Omega_{n}^{c}\right) \\
& \leq n \sum_{2 \leq k \leq(\log n)^{2}} \mathbb{P}\left(\frac{S_{k}^{+}}{\left.\sqrt{k-S_{k}^{+}} \geq 0.9 \varepsilon \sqrt{n}\right)+\mathbb{P}\left(\Omega_{n}^{c}\right)}\right. \\
& \leq n \sum_{2 \leq k \leq(\log n)^{2}} \exp \left[-k I^{+}\left\{g^{+}\left(\frac{0.9 \varepsilon \sqrt{n}}{\sqrt{k}}\right\}\right]\right\}+\mathbb{P}\left(\Omega_{n}^{c}\right) \\
& \leq n \sum_{2 \leq k \leq(\log n)^{2}} \exp \left[k g^{+}\left(\frac{0.9 \varepsilon \sqrt{n}}{\sqrt{k}}\right)+k \log \left\{1-g^{+}\left(\frac{0.9 \varepsilon \sqrt{n}}{\sqrt{k}}\right)\right\}\right]+\mathbb{P}\left(\Omega_{n}^{c}\right) .
\end{aligned}
$$

As $a \rightarrow \infty, 0.9 \varepsilon \sqrt{n} / \sqrt{k} \rightarrow \infty$ and $g^{+}(a) \uparrow 1$. In addition,

$$
1-g^{+}(a)=1-\frac{a\left(\sqrt{a^{2}+4}-a\right)}{2}=1-\frac{2 a}{\sqrt{a^{2}+4}+a}=\frac{\sqrt{a^{2}+4}-a}{\sqrt{a^{2}+4}+a}=\frac{4}{\left(\sqrt{a^{2}+4}+a\right)^{2}} .
$$

Note that

$$
\frac{0.9}{a^{2}} \leq \frac{4}{\left(\sqrt{a^{2}+4}+a\right)^{2}} \leq \frac{1}{a^{2}},
$$

when $a$ is large enough. Therefore, when $n$ is sufficiently large,

$$
\begin{aligned}
\mathbb{P}\left(\tilde{M}_{n}^{+}\left(2,(\log n)^{2}\right) \geq \varepsilon \sqrt{n}\right) & \leq n \sum_{2 \leq k \leq(\log n)^{2}} \exp \left\{k-k \log \left(\frac{0.9 \varepsilon n}{k}\right)\right\} \\
& \leq n \sum_{2 \leq k \leq(\log n)^{2}} \exp (-0.9 k \log n) \\
& \leq n \sum_{2 \leq k \leq(\log n)^{2}} \exp (-1.8 \log n) \rightarrow 0,
\end{aligned}
$$

where the last inequality uses that $k \geq 2$.

When $(\log n)^{2} \leq j-i \leq n-(\log n)^{2}$, by Theorem 1 and Theorem 2, we have

$$
\begin{gathered}
U_{(j)}-U_{(i)} \leq \frac{j-i}{n}+\frac{1.01 \log n}{\sqrt{n}} w_{i, j}^{n}, \\
1-\left(U_{(j)}-U_{(i)}\right) \geq 1-\frac{j-i}{n}-\frac{1.01 \log n}{\sqrt{n}} w_{i, j}^{n}, \\
U_{(j)}-U_{(i)} \geq \frac{j-i}{n}-\frac{1.01 \log n}{\sqrt{n}} w_{i, j}^{n},
\end{gathered}
$$

and

$$
1-\left(U_{(j)}-U_{(i)}\right) \leq 1-\frac{j-i}{n}+\frac{1.01 \log n}{\sqrt{n}} w_{i, j}^{n},
$$


with probability tending to one. Together, (132) and (134) lead to

$$
\left|\frac{n\left(U_{(j)}-U_{(i)}\right)}{j-i}\right|=O_{P}(1),
$$

uniformly in $(i, j)$ satisfying $j-i \geq(\log n)^{2}$. (133) and (135) imply

$$
\left|\frac{1-\left(U_{(j)}-U_{(i)}\right)}{1-(j-i) / n}\right|=O_{P}(1) .
$$

These, combined with the definitions of $M_{n}^{+}$and $\tilde{M}_{n}^{+}$, imply

$$
\tilde{M}_{n}^{+}\left\{(\log n)^{2}, n-(\log n)^{2}\right\} \asymp_{P} M_{n}^{+}\left\{(\log n)^{2}, n-(\log n)^{2}\right\} .
$$

By Theorem 1, it follows that for any $\varepsilon>0$,

$$
\lim _{n \rightarrow \infty} \mathbb{P}\left[\tilde{M}_{n}^{+}\left\{(\log n)^{2}, n-(\log n)^{2}\right\} \geq \varepsilon \sqrt{n}\right]=0 .
$$

Finally, when $n-(\log n)^{2} \leq j-i \leq n$, define $j^{\prime}=n-j$ and thus $U_{\left(j^{\prime}+1\right)}^{\prime}=1-U_{\left(n+1-j^{\prime}-1\right)}=1-U_{(j)}$. A simple change of indices gives

$$
\begin{aligned}
& \tilde{M}_{n}^{+}\left(n-(\log n)^{2}, n\right) \\
& =\max _{\substack{0 \leq i<j \leq n \\
n-(\log n)^{2} \leq j-i \leq n}} \frac{j-i-n\left(U_{(j)}-U_{(i)}\right)}{\sqrt{n\left(U_{(j)}-U_{(i)}\right)\left(1-\left(U_{(j)}-U_{(i)}\right)\right)}} \\
& =\max _{\substack{i, j^{\prime} \geq 0 \\
i+j^{\prime} \leq(\log n)^{2}}} \frac{n U_{\left(j^{\prime}+1\right)}^{\prime}-\left(j^{\prime}+1\right)+n U_{(i)}-i}{\sqrt{n\left(U_{(i)}+U_{\left(j^{\prime}+1\right)}^{\prime}\right)\left(1-U_{(i)}-U_{\left(j^{\prime}+1\right)}^{\prime}\right)}} \\
& =\max _{\substack{i, j \geq 0 \\
1 \leq i+j \leq(\log n)^{2}}} \frac{n U_{(i)}-i+n U_{(j)}^{\prime}-j}{\sqrt{n\left(U_{(i)}+U_{(j)}^{\prime}\right)\left(1-U_{(i)}-U_{(j)}^{\prime}\right)}}+O_{P}(1) .
\end{aligned}
$$

Notice that when $i, j \geq 0$ and $1 \leq i+j \leq(\log n)^{2}$,

$$
1-U_{(i)}-U_{(j)}^{\prime}>1-2 \max _{0 \leq i \leq(\log n)^{2}} U_{(i)}>0.5,
$$

with probability tending to one, which can be seen by a simple application of Kolmogorov's Theorem. By a similar speech when proving $R_{5}$ in the proof of Theorem 1.

$$
\begin{aligned}
& \mathbb{P}\left(\max _{\substack{i, j \geq 0 \\
1 \leq i+j \leq(\log n)^{2}}} \frac{n U_{(i)}-i+n U_{(j)}^{\prime}-j}{\sqrt{n\left(U_{(i)}+U_{(j)}^{\prime}\right)\left(1-U_{(i)}-U_{(j)}^{\prime}\right)}} \geq \varepsilon \sqrt{n}\right) \\
& \leq \mathbb{P}\left(\max _{\substack{i, j \geq 0 \\
1 \leq i+j \leq(\log n)^{2}}} \frac{n U_{(i)}-i+n U_{(j)}^{\prime}-j}{\sqrt{n\left(U_{(i)}+U_{(j)}^{\prime}\right)}} \geq 0.5 \varepsilon \sqrt{n}\right) \\
& \leq 2 \mathbb{P}\left(\max _{0 \leq i \leq(\log n)^{2}} \frac{n U_{(i)}-i}{\sqrt{n U_{(i)}}} \geq 0.25 \varepsilon \sqrt{n}\right) \\
& \leq 2 \mathbb{P}\left(\max _{0 \leq i \leq(\log n)^{2}} \frac{n U_{(i)}-i}{\sqrt{n U_{(i)}\left(1-U_{(i)}\right)}} \geq 0.25 \varepsilon \sqrt{n}\right) \\
& \rightarrow 0,
\end{aligned}
$$

where the last line again follows from Eicker [13]. These eventually establish the proof of (27). 


\subsubsection{Proof of (28)}

\section{The roadmap of our proof.}

To derive the asymptotic distribution, we first focus on the most contributed part, i.e., those with length $j-i=l_{n} \sim a \log ^{3} n$ for $a>0$. Define

$$
u_{n}=u_{n}(\tau):=\sqrt{2 \log n}\left(1+\frac{-3 \log \log n+2 \tau}{4 \log n}\right) .
$$

For any two constants $0<A_{1}<A_{2}<\infty$, define $l_{n}^{-}=A_{1} \log ^{3} n$ and $l_{n}^{+}=A_{2} \log ^{3} n$. We prove

$$
\lim _{n \rightarrow \infty} \mathbb{P}\left\{\tilde{M}_{n}^{+}\left(l_{n}^{-}, l_{n}^{+}\right) \leq u_{n}\right\}=\exp \left\{-e^{-\tau} \int_{A_{1}}^{A_{2}} \Lambda_{1}(a) d a\right\} .
$$

It turns out that to prove (147), within that region, it suffices to focus on

$$
\tilde{Z}_{i, j}^{+}:=\frac{S_{j}^{+}-S_{i}^{+}}{\sqrt{j-i-\left(S_{j}^{+}-S_{i}^{+}\right)}},
$$

instead, up to restricting on subset $\Omega_{n}$ defined in (81). Write

$$
\tilde{Z}_{n}^{+}(k, l)=\max _{0 \leq i<j \leq n: k \leq j-i \leq l} \tilde{Z}_{i, j}^{+},
$$

and

$$
\tilde{Z}_{n}^{+}=\tilde{Z}_{n}^{+}(1, n)
$$

We will use Lemma 5 to show that

$$
\mathcal{Q}_{n}:=\mathbb{P}\left(\max _{(i, j) \in \mathbb{T}_{B q_{n}}\left(x, x+l_{n}\right)} \tilde{Z}_{i, j}^{+} \geq u_{n}\right) \sim P_{n}(0)\left\{1+H^{2}\left(\frac{B}{a}\right)\right\},
$$

where $B \geq 1$ is an integer and the quantities $P_{n}(0), H(x), q_{n}$ will be specified later. Next, with a domain $\mathbb{J}_{n}(z)$ (to be specified) larger than $\mathbb{T}_{B q_{n}}$, we will show that

$$
\mathbb{P}\left(\max _{(i, j) \in \mathbb{J}_{n}(z)} \tilde{Z}_{i, j}^{+} \geq u_{n}\right) \sim e^{-\tau} \frac{w_{n}}{n} \int_{A_{1}}^{A_{2}} \Lambda_{1}(a) d a,
$$

which no longer depends on $B$, with $\Lambda_{1}(a)$ defined in the theorem part. This enables us to apply Poisson limit theorem in [3] to get

$$
\lim _{n \rightarrow \infty} \mathbb{P}\left\{\tilde{Z}_{n}^{+}\left(l_{n}^{-}, l_{n}^{+}\right) \leq u_{n}\right\}=\exp \left\{-e^{-\tau} \int_{A_{1}}^{A_{2}} \Lambda_{1}(a) d a\right\} .
$$

The final step will be showing that the region beyond $A_{2}(\log n)^{3}$ is negligible, that is,

$$
\limsup _{A_{2} \rightarrow \infty} \limsup _{n \rightarrow \infty} \mathbb{P}\left\{\tilde{M}_{n}^{+}\left(l_{n}^{+}, n\right) \geq u_{n}\right\}=0 .
$$

Therefore setting $A_{1}=A$ and letting $A_{2} \rightarrow \infty$ yield (28).

We first argue why we can focus on (53) instead when $j-i \asymp \log ^{3} n$. Note that (128) and (129) continue to hold when $j-i \asymp(\log n)^{3}$. Hence,

$$
\tilde{M}_{n}^{+}\left(l_{n}^{-}, l_{n}^{+}\right)=\left\{1+O_{P}(1 / \sqrt{n})\right\} \tilde{Z}_{n}^{+}\left(l_{n}^{-}, l_{n}^{+}\right)+O_{P}(1 / \sqrt{n}),
$$


which implies

$$
\mathbb{P}\left\{\tilde{Z}_{n}^{+}\left(l_{n}^{-}, l_{n}^{+}\right) \leq u_{n}(\tau-\varepsilon)\right\} \leq \mathbb{P}\left\{\tilde{M}_{n}^{+}\left(l_{n}^{-}, l_{n}^{+}\right) \leq u_{n}(\tau)\right\} \leq \mathbb{P}\left\{\tilde{Z}_{n}^{+}\left(l_{n}^{-}, l_{n}^{+}\right) \leq u_{n}(\tau+\varepsilon)\right\},
$$

for any $\varepsilon>0$. If we had established (153), taking $\varepsilon \rightarrow 0$ would yield (147). Now we turn to the mainstream of the proof.

Proof of (151). We will prove this following a similar strategy as in Kabluchko and Wang [25]. Necessary adjustments are still needed since Kabluchko and Wang [25] focused on $Z_{i, j}^{+}$while we are dealing with $\tilde{Z}_{i, j}^{+}$. We will present the parts that need to be adjusted and refer to their results when nothing needs to be changed.

First we work on $\mathcal{Q}_{n}$. For any $\tau \in \mathbb{R}$ and $a \geq 0$, let $l_{n}=a(\log n)^{3}$ and define

$$
P_{n}(s)=\mathbb{P}\left(\frac{S_{l_{n}}^{+}}{\sqrt{l_{n}-S_{l_{n}}^{+}}} \geq u_{n}-\frac{s}{u_{n}}\right) .
$$

Define

$$
b_{n}:=\frac{u_{n}-s / u_{n}}{\sqrt{l_{n}}}
$$

for ease of notation. Since $u_{n}^{3} \propto \sqrt{l_{n}}$ and $b_{n} \sim \sqrt{2 / a} / \log n \rightarrow 0$, for fixed $s>0$ with sufficiently large $n$, with the transformation (57), Lemma 1 and Taylor's expansion

$$
\begin{aligned}
P_{n}(s) & =\mathbb{P}\left\{\frac{S_{l_{n}}^{+}}{\sqrt{l_{n}}} \geq \sqrt{l_{n}} g^{+}\left(b_{n}\right)\right\} \\
& \sim \frac{1}{\sqrt{2 \pi} u_{n}} \exp \left\{-\frac{\left(u_{n}-s / u_{n}\right)^{2}}{2} \frac{2 I^{+}\left(g^{+}\left(b_{n}\right)\right)}{b_{n}^{2}}\right\} \\
& =\frac{1}{\sqrt{2 \pi} u_{n}} \exp \left\{-\frac{\left(u_{n}-s / u_{n}\right)^{2}}{2}\left(1-\frac{1}{3} b_{n}\right)+o(1)\right\} \\
& \sim \frac{1}{2 \sqrt{\pi}} e^{s+\frac{\sqrt{2}}{3} a^{-1 / 2}} \frac{e^{-\tau} \log n}{n} .
\end{aligned}
$$

Recall that $\mathbb{T}_{r}(x, y)$ is defined in (48). Define $q_{n}=(\log n)^{2}$. By the same techniques in the proof of Lemma 7 we have

$$
\begin{aligned}
\mathcal{Q}_{n} & =\mathbb{P}\left(\max _{(i, j) \in \mathbb{T}_{B q_{n}}\left(x, x+l_{n}\right)} \tilde{Z}_{i, j}^{+} \geq u_{n}\right) \\
& =\mathbb{P}\left[\max _{(i, j) \in \mathbb{T}_{B q_{n}}\left(x, x+l_{n}\right)}\left\{S_{j}^{+}-S_{i}^{+}-(j-i) g^{+}\left(\frac{u_{n}}{j-i}\right)\right\} \geq 0\right] \\
& =\mathbb{P}\left[\max _{0 \leq k_{1}, k_{2} \leq B q_{n}}\left\{S_{k_{1}}^{(1)+}+S_{k_{2}}^{(2)+}-\left(l_{n}+k_{1}+k_{2}\right) g^{+}\left(\frac{u_{n}}{l_{n}+k_{1}+k_{2}}\right)\right\}+S_{l_{n}}^{+} \geq 0\right] \\
& =P_{n}(0)\left\{1+\int_{0}^{\infty} G_{n}(s) d \nu_{n}(s)\right\},
\end{aligned}
$$

where $P_{n}(s)$ defined in (156) is actually the probability distribution of $V_{l_{n}, u_{n}}$, defined in (63). Therein

$$
\begin{aligned}
G_{n}(s):=\mathbb{P}\left[\max _{0 \leq k_{1}, k_{2} \leq B q_{n}}\left\{S_{k_{1}}^{(1)+}+S_{k_{2}}^{(2)+}-\left(l_{n}+k_{1}+k_{2}\right) g^{+}\left(\frac{u_{n}}{\sqrt{l_{n}+k_{1}+k_{2}}}\right)\right\}\right. \\
\left.+l_{n} \cdot g^{+}\left(\frac{u_{n}-s / u_{n}}{\sqrt{l_{n}}}\right) \geq 0\right]
\end{aligned}
$$


and

$$
\nu_{n}(\cdot):=P_{n}(\cdot) / P_{n}(0) .
$$

It is immediate that the first and second conditions in Lemma 5 hold by directly mimicking the details in the proof of Lemma 4.3 in [25], that is, for any fixed $s>0$ and any sequence $s_{n} \rightarrow s$,

$$
\lim _{n \rightarrow \infty} G_{n}\left(s_{n}\right)=\mathbb{P}\left(M_{1}+M_{2} \geq s\right)
$$

and

$$
\lim _{n \rightarrow \infty} \nu_{n}([0, s))=\lim _{n \rightarrow \infty} \frac{P_{n}(s)}{P_{n}(0)}=e^{s} .
$$

$M_{1}$ and $M_{2}$ are independent copies with the same distribution as

$$
M=\sup _{t \in\left[0, a^{-1} B\right]}\{\sqrt{2} W(t)-t\}
$$

where $W(t)$ is a standard Brownian motion (similar but more detailed arguments can be found in the proof of lemma 4.3 in [24]). To verify the third condition in Lemma 5, we need to bound the integral $\int_{0}^{\infty} G_{n}(s) d \nu_{n}(s)$ from above. This can be immediately completed by using Lemma 7. Hence applying Lemma 5 completes the proof of (151), where

$$
H(x):=\mathbb{E}\left\{\sup _{t \in[0, x]} e^{\sqrt{2} W(t)-t}\right\}, x>0,
$$

therein.

Proof of (152). Define $w_{n}=(\log n)^{3}$. For $z \in \mathbb{Z}$, define

$$
\mathbb{J}_{n}(z)=\left\{(i, j) \in \mathbb{I}: z \leq i<z+w_{n}, j-i \in\left[l_{n}^{-}, l_{n}^{+}\right]\right\} .
$$

To derive the rate of $\mathbb{P}\left(\max _{(i, j) \in \mathbb{J}_{n}(z)} \tilde{Z}_{i, j}^{+} \geq u_{n}\right)$, by translation invariance we may take $z=0$. Let $\delta_{n}$ be a real sequence satisfying $\delta_{n}=o\left(w_{n}\right)$ and $q_{n}=o\left(\delta_{n}\right)$, e.g. $\delta_{n}=(\log n)^{2.5}$. For $B \in \mathbb{N}$, we introduce the following two-dimensional discrete grids with mesh size $B q_{n}$ :

$$
\begin{gathered}
\mathcal{J}_{n}(B)=\left\{(x, y) \in B q_{n} \mathbb{Z} \times B q_{n} \mathbb{Z}: x \in\left[-\delta_{n}, w_{n}+\delta_{n}\right], y-x \in\left[l_{n}^{-}-\delta_{n}, l_{n}^{+}+\delta_{n}\right]\right\}, \\
\mathcal{J}_{n}^{\prime}(B)=\left\{(x, y) \in B q_{n} \mathbb{Z} \times B q_{n} \mathbb{Z}: x \in\left[\delta_{n}, w_{n}-\delta_{n}\right], y-x \in\left[l_{n}^{-}+\delta_{n}, l_{n}^{+}-\delta_{n}\right]\right\} .
\end{gathered}
$$

By Bonferroni inequality,

$$
S_{n}^{\prime}(B)-S_{n}^{\prime \prime}(B) \leq \mathbb{P}\left(\max _{(i, j) \in \mathbb{J}_{n}(0)} \tilde{Z}_{i, j}^{+} \geq u_{n}\right) \leq S_{n}(B)
$$

where

$$
\begin{aligned}
& S_{n}(B)=\sum_{(x, y) \in \mathcal{J}_{n}(B)} \mathbb{P}\left(\max _{(i, j) \in \mathbb{T}_{B q_{n}}(x, y)} \tilde{Z}_{i, j}^{+} \geq u_{n}\right), \\
& S_{n}^{\prime}(B)=\sum_{(x, y) \in \mathcal{J}_{n}^{\prime}(B)} \mathbb{P}\left(\max _{(i, j) \in \mathbb{T}_{B q_{n}}(x, y)} \tilde{Z}_{i, j}^{+} \geq u_{n}\right),
\end{aligned}
$$

and

$$
S_{n}^{\prime \prime}(B)=\sum_{\left(x_{1}, y_{1}\right),\left(x_{2}, y_{2}\right)} \mathbb{P}\left(\max _{(i, j) \in \mathbb{T}_{B q_{n}}\left(x_{1}, y_{1}\right)} \tilde{Z}_{i, j}^{+} \geq u_{n}, \max _{(i, j) \in \mathbb{T}_{B q_{n}}\left(x_{2}, y_{2}\right)} \tilde{Z}_{i, j}^{+} \geq u_{n}\right)
$$


where the summation is taken over $\left(x_{1}, y_{1}\right) \neq\left(x_{2}, y_{2}\right) \in \mathcal{J}_{n}^{\prime}(B)$. As long as we can show

$$
\begin{aligned}
& \lim _{B \rightarrow \infty} \limsup _{n \rightarrow \infty} n w_{n}^{-1} S_{n}(B) \leq e^{-\tau} \int_{A_{1}}^{A_{2}} \Lambda_{1}(a) d a, \\
& \lim _{B \rightarrow \infty} \liminf _{n \rightarrow \infty} n w_{n}^{-1} S_{n}^{\prime}(B) \geq e^{-\tau} \int_{A_{1}}^{A_{2}} \Lambda_{1}(a) d a,
\end{aligned}
$$

and

$$
\lim _{B \rightarrow \infty} \limsup _{n \rightarrow \infty} n w_{n}^{-1} S_{n}^{\prime \prime}(B)=0
$$

(152) will follow immediately. The proof of (172) is almost identical to that of (171), so we only focus on proving (171) based on the dominated convergence theorem. Define

$$
\mathcal{L}_{n}(B)=B q_{n} \mathbb{Z} \cap\left[l_{n}^{-}-\delta_{n}, l_{n}^{+}+\delta_{n}\right]
$$

such that $\left|\mathcal{L}_{n}(B)\right| \sim\left(A_{2}-A_{1}\right)(\log n) / B$. Since the probability on the right-hand side of (168) depends only on $l:=y-x$, by translation invariance we have

$$
S_{n}(B) \leq \frac{w_{n}+\delta_{n}}{B q_{n}} \sum_{l \in \mathcal{L}_{n}(B)} \mathbb{P}\left(\max _{(i, j) \in T_{B q_{n}}(0, l)} \tilde{Z}_{i, j}^{+} \geq u_{n}\right) .
$$

Next we apply (151) to bound each probability with $l$ fixed and replace $\left(B q_{n}\right)^{-1} \sum_{l \in \mathcal{L}_{n}(B)}$ by an integral as $n \rightarrow \infty$. By (151) and (158),

$$
\lambda_{n, B}(a):=\frac{n}{\log n} \mathbb{P}\left(\max _{(i, j) \in T_{B q_{n}}\left(0, l_{n, B}(a)\right)} \tilde{Z}_{i, j}^{+} \geq u_{n}\right) \rightarrow \frac{1}{2 \sqrt{\pi}} e^{\frac{\sqrt{2}}{3} a^{-1 / 2}-\tau}\left\{1+H^{2}\left(\frac{B}{a}\right)\right\},
$$

as $n \rightarrow \infty$, where

$$
l_{n, B}(a)=\max \left\{l \in B q_{n} \mathbb{Z}: l \leq a w_{n}\right\} .
$$

The function $\lambda_{n, B}(a)$ takes constant values on sub-intervals with widths $B q_{n} / w_{n}=B / \log n$. It follows that

$$
S_{n}(B) \leq \frac{w_{n}+\delta_{n}}{B^{2} n} \sum_{l \in \mathcal{L}_{n}(B)} \frac{B \lambda_{n, B}(a)}{\log n}=\frac{w_{n}+\delta_{n}}{B^{2} n} \int_{A_{1}-\frac{2 \delta_{n}}{w_{n}}}^{A_{2}+\frac{2 \delta_{n}}{w_{n}}} \lambda_{n, B}(a) d a
$$

From Lemma 7 we can upper bound the integrand $\lambda_{n, B}(a)$ by an integrable function that is independent of $n$. Therefore, applying Fatou's lemma on lim sup gives

$$
\limsup _{n \rightarrow \infty} n w_{n}^{-1} S_{n}(B) \leq e^{-\tau} \int_{A_{1}}^{A_{2}} \frac{a^{2} \Lambda_{1}(a)}{B^{2}}\left\{1+H^{2}\left(\frac{B}{a}\right)\right\} d a
$$

This result holds for any $B \in \mathbb{N}$. Note that $\lim _{B \rightarrow \infty} H(B) / B=1$. Letting $B \rightarrow \infty$, we arrive at (171).

To prove (173), we bound $S_{n}^{\prime \prime}(B)$ by similar quantities of $Z_{i, j}^{+}$, which allows us to use results in Kabluchko and Wang [25] immediately. For any interval $(x, y)$ define the event

$$
E_{n}(x, y)=\left\{\max _{(i, j) \in \mathbb{T}_{B q_{n}}(x, y)} \tilde{Z}_{i, j}^{+} \geq u_{n}\right\}
$$


Note that

$$
\frac{g^{+}(x)}{x}=\frac{1}{2}\left(\sqrt{x^{2}+4}-x\right) \geq 1-\frac{x}{2}, \text { when } x \rightarrow 0 .
$$

When $y-x \propto(\log n)^{3}, u_{n} /(y-x) \propto 1 /(\log n)$,

$$
\begin{aligned}
E_{n}(x, y) & =\left\{\max _{0 \leq l_{1}, l_{2} \leq B q_{n}}\left\{S_{y+l_{2}}^{+}-S_{x-l_{1}}^{+}-\left(y-x+l_{1}+l_{2}\right) g^{+}\left(\frac{u_{n}}{\sqrt{y-x+l_{1}+l_{2}}}\right)\right\} \geq 0\right\} \\
& \subset\left\{\max _{0 \leq l_{1}, l_{2} \leq B q_{n}} \frac{S_{y+l_{2}}^{+}-S_{x-l_{1}}^{+}}{\sqrt{y-x+l_{1}+l_{2}}} \geq \sqrt{y-x+l_{1}+l_{2}} g^{+}\left(\frac{u_{n}}{\sqrt{y-x+l_{1}+l_{2}}}\right)\right\} \\
& \subset\left\{\max _{(i, j) \in \mathbb{T}_{B q_{n}}(x, y)} Z_{i, j}^{+} \geq u_{n}(\tau)\left(1-\frac{u_{n}}{2 \sqrt{y-x+l_{1}+l_{2}}}\right)\right\} \\
& \subset\left\{\max _{(i, j) \in \mathbb{T}_{B q_{n}}(x, y)} Z_{i, j}^{+} \geq u_{n}(\tau-0.1)\right\} .
\end{aligned}
$$

Therefore,

$$
\begin{aligned}
& \mathbb{P}\left\{E_{n}\left(i_{1}, j_{1}\right) \cap E_{n}\left(i_{2}, j_{2}\right)\right\} \\
& \leq \mathbb{P}\left[\left\{\max _{(i, j) \in \mathbb{T}_{B q_{n}}\left(i_{1}, j_{1}\right)} Z_{i, j}^{+} \geq u_{n}(\tau-0.1)\right\} \bigcap\left\{\max _{(i, j) \in \mathbb{T}_{B q_{n}}\left(i_{2}, j_{2}\right)} Z_{i, j}^{+} \geq u_{n}(\tau-0.1)\right\}\right] .
\end{aligned}
$$

This allows us to work on $Z_{i, j}^{+}$instead. Directly applying Lemma 4.12, Lemma 4.14, Lemma 4.15 and Lemma 4.16 in [25] yields (173).

Proof of (153). We will temporarily adopt the notations in Arratia et al. [3]. Define

$$
I=\left\{\alpha \in \mathbb{N}: \alpha w_{n} \leq n\right\},
$$

which implies $|I| \leq n / w_{n}$. For any $\alpha \in I$, define

$$
\begin{gathered}
X_{\alpha}=\mathbb{1}\left\{\max _{(i, j) \in \mathbb{J}_{n}\left(\alpha w_{n}\right)} \tilde{Z}_{i, j}^{+} \geq u_{n}\right\}, \\
p_{\alpha}=\mathbb{P}\left(X_{\alpha}\right),
\end{gathered}
$$

and

$$
B_{\alpha}=\left\{\beta \in I:\left|(\beta-\alpha) w_{n}\right| \leq l_{n}^{+}+w_{n}\right\} .
$$

Hence $\left|B_{\alpha}\right| \leq A_{2}+1$. To apply Theorem 1 in [3], we need to show that

$$
\begin{gathered}
b_{1}:=\sum_{\alpha \in I} \sum_{\beta \in B_{\alpha}} p_{\alpha} p_{\beta}, \\
b_{2}:=\sum_{\alpha \in I} \sum_{\alpha \neq \beta \in B_{\alpha}} p_{\alpha \beta}, \text { where } p_{\alpha \beta}:=\mathbb{E}\left(X_{\alpha} X_{\beta}\right),
\end{gathered}
$$

and

$$
b_{3}^{\prime}:=\sum_{\alpha \in I} s_{\alpha}^{\prime}
$$

therein vanish as $n \rightarrow \infty$, where

$$
s_{\alpha}^{\prime}:=\mathbb{E}\left|\mathbb{E}\left(X_{\alpha}-p_{\alpha} \mid \sum_{\beta \in I-B_{\alpha}} X_{\beta}\right)\right|
$$


By the definition of $B_{\alpha}, X_{\alpha}-p_{\alpha}$ and $\sum_{\beta \in I-B_{\alpha}} X_{\beta}$ are independent. Hence $s_{\alpha}^{\prime}=0$, so is $b_{3}^{\prime}$. It follows from (152) that

$$
b_{1} \sim|I|\left|B_{\alpha}\right| p_{\alpha} p_{\beta} \rightarrow 0 .
$$

With slight modification on (152),

$$
\mathbb{P}\left(\max _{(i, j) \in \mathbb{J}_{n}\left(\alpha w_{n}\right) \cup \mathbb{J}_{n}\left(\beta w_{n}\right)} \tilde{Z}_{i, j}^{+} \geq u_{n}\right) \sim e^{-\tau} \frac{2 w_{n}}{n} \int_{A_{1}}^{A_{2}} \Lambda_{1}(a) d a .
$$

This, together with (152), implies

$$
p_{\alpha \beta}=\mathbb{P}\left(\max _{(i, j) \in \mathbb{J}_{n}\left(\alpha w_{n}\right)} \tilde{Z}_{i, j}^{+} \geq u_{n}, \max _{(i, j) \in \mathbb{J}_{n}\left(\beta w_{n}\right)} \tilde{Z}_{i, j}^{+} \geq u_{n}\right)=o\left(\frac{w_{n}}{n}\right) .
$$

Thus,

$$
b_{2} \leq|I|\left|B_{\alpha}\right| \max _{\alpha \neq \beta} p_{\alpha \beta} \rightarrow 0
$$

Now, by Theorem 1 in [3],

$$
\lim _{n \rightarrow \infty} \mathbb{P}\left\{\tilde{Z}_{n}^{+}\left(l_{n}^{-}, l_{n}^{+}\right) \leq u_{n}\right\}=\lim _{n \rightarrow \infty} \mathbb{P}\left(\sum_{\alpha \in I} X_{\alpha}=0\right)=e^{-\lambda}
$$

where

$$
\lambda=\sum_{\alpha \in I} p_{\alpha} \rightarrow e^{-\tau} \int_{A_{1}}^{A_{2}} \Lambda_{1}(a) d a
$$

Therefore,

$$
\lim _{n \rightarrow \infty} \mathbb{P}\left\{\tilde{M}_{n}^{+}\left(l_{n}^{-}, l_{n}^{+}\right) \leq u_{n}\right\}=\exp \left(-e^{-\tau} \int_{A_{1}}^{A_{2}} \Lambda_{1}(a) d a\right),
$$

by the statement in the beginning of our proof.

Proof of (154). Divide $\left(l_{n}^{+}, n\right]$ into $\left(l_{n}^{+},(\log n)^{4}\right],\left((\log n)^{4}, n-(\log n)^{4}\right]$ and $\left(n-(\log n)^{4}, n\right]$. Within the first region, for any $k \in \mathbb{N}$, any pair $(i, j)$ with length $2^{k}(\log n)^{3} \leq j-i \leq 2^{k+1}(\log n)^{3}$ can be covered by the union of at most $2^{-k} n / \log n$ disjoint discrete squares of the form $\mathbb{T}_{2^{k}(\log n)^{2}}(x, x+$ $j-i)$. By (133),

$$
1-\left(U_{(j)}-U_{(i)}\right) \geq 1-1.1(\log n)^{4} / n,
$$

with probability tending to one. With these facts, by the union bound and Lemma 7 ,

$$
\begin{aligned}
& \mathbb{P}\left\{\tilde{M}_{n}^{+}\left(l_{n}^{+},(\log n)^{4}\right) \geq u_{n}\right\} \\
& \leq \mathbb{P}\left\{\max _{k: \log _{2} A_{2} \leq k \leq \log _{2}(\log n)} \tilde{M}_{n}^{+}\left(2^{k}(\log n)^{3}, 2^{k+1}(\log n)^{3}\right) \geq u_{n}\right\} \\
& \leq \mathbb{P}\left\{\tilde{m a x}_{k: \log _{2} A_{2} \leq k \leq \log _{2}(\log n)} \tilde{Z}_{n}^{+}\left(2^{k}(\log n)^{3}, 2^{k+1}(\log n)^{3}\right) \geq u_{n}(\tau-0.1)\right\} \\
& \leq \sum_{k \geq \log _{2} A_{2}} 2^{-k} \frac{n}{\log n} \mathbb{P}\left\{\tilde{Z}_{(i, j) \in T_{2^{k}(\log n)^{2}}\left(0,2^{k+1}(\log n)^{3}\right)}^{+} \geq u_{n}(\tau-0.1)\right\}+\mathbb{P}\left(\Omega_{n}^{c}\right) \\
& \leq C \sum_{k \geq \log _{2} A_{2}} 2^{-k}+\mathbb{P}\left(\Omega_{n}^{c}\right) .
\end{aligned}
$$

Taking $\lim \sup _{n \rightarrow \infty}$ and letting $A_{2} \rightarrow \infty$ gives the desired result. 
In the meantime, on $\left((\log n)^{4}, n-(\log n)^{4}\right]$, a finer examination of (132) and (134) yields

$$
\left|\frac{n\left(U_{(j)}-U_{(i)}\right)}{j-i}-1\right|=O_{p}\left(\frac{1}{\log n}\right) .
$$

(133) and (135) imply

$$
\left|\frac{1-\left(U_{(j)}-U_{(i)}\right)}{1-(j-i) / n}-1\right|=O_{p}\left(\frac{1}{\log n}\right)
$$

Therefore,

$$
\mathbb{P}\left\{\tilde{M}_{n}^{+}\left((\log n)^{4}, n-(\log n)^{4}\right) \geq u_{n}\right\} \leq \mathbb{P}\left\{M_{n}^{+}\left(l_{n}^{+},(\log n)^{4}\right) \geq u_{n}(\tau-0.1)\right\} \rightarrow 0,
$$

by Theorem 1 ,

The proof of the region $\left(n-(\log n)^{4}, n\right]$ is immediate by following the proof for (145), which we omit here.

\subsection{Proof of Theorem 4}

Define

$$
\tilde{Z}_{i, j}^{-}:=\frac{S_{j}^{-}-S_{i}^{-}}{\sqrt{j-i+S_{j}^{-}-S_{i}^{-}}}
$$

and

$$
\begin{gathered}
g^{-}(a):=\frac{1}{2}\left(a \sqrt{a^{2}+4}+a^{2}\right) . \\
I^{-}\left(g^{-}(s)\right) \geq s^{2} / 2 .
\end{gathered}
$$

The theorem follows immediately after showing that

$$
\limsup _{n \rightarrow \infty} \mathbb{P}\left(\tilde{M}_{n}^{-} \geq \varepsilon \sqrt{n}\right)=0
$$

for any $\varepsilon>0$. This can be proved similarly by dividing the regions, transforming the statistic $\tilde{M}_{i, j}^{-}$ into $\tilde{Z}_{i, j}^{-}$, combined with (202). We omit the detail here.

\section{Acknowledgements}

Andrew Ying was partially supported by the Achievement Rewards for College Scientists (ARCS) Scholarship. The authors strongly thanks for Professor Ery Arias-Castro for building up the introduction and providing the motivation. The authors would also like to thank for Professor Qi-Man Shao, Professor Xiao Fang, Professor Hock Peng Chan, and Professor David O. Siegmund for stimulating discussions and pointers to the literature.

\section{References}

[1] Aldous, D. (2013). Probability approximations via the Poisson clumping heuristic, Volume 77. Springer Science \& Business Media.

[2] Anderson, T. W. and D. A. Darling (1952). Asymptotic theory of certain "goodness of fit" criteria based on stochastic processes. The annals of mathematical statistics, 193-212. 
[3] Arratia, R., L. Goldstein, and L. Gordon (1989). Two moments suffice for poisson approximations: the chen-stein method. The Annals of Probability 17(1), 9-25.

[4] Bahadur, R. R. and R. R. Rao (1960). On deviations of the sample mean. Ann. Math. Statist 31(4), 1015-1027.

[5] Berg, W. (1945). Aggregates in one-and two-dimensional random distributions. The London, Edinburgh, and Dublin Philosophical Magazine and Journal of Science 36(256), 337-346.

[6] Berk, R. H. and D. H. Jones (1979). Goodness-of-fit test statistics that dominate the kolmogorov statistics. Zeitschrift für Wahrscheinlichkeitstheorie und verwandte Gebiete 47(1), 47-59.

[7] Besag, J. and J. Newell (1991). The detection of clusters in rare diseases. Journal of the Royal Statistical Society: Series A (Statistics in Society) 154(1), 143-155.

[8] Chan, H. P. and T. L. Lai (2006). Maxima of asymptotically gaussian random fields and moderate deviation approximations to boundary crossing probabilities of sums of random variables with multidimensional indices. The Annals of Probability 34(1), 80-121.

[9] Cramér, H. (1938). Les sommes et les fonctions de variables aléatoires, Volume 736. Hermann.

[10] Darling, D. and P. Erdös (1956). A limit theorem for the maximum of normalized sums of independent random variables. Duke Mathematical Journal 23(1), 143-155.

[11] Deheuvels, P., L. Devroye, J. Lynch, et al. (1986). Exact convergence rate in the limit theorems of erdos-renyi and shepp. The Annals of Probability 14(1), 209-223.

[12] Dumbgen, L. and V. G. Spokoiny (2001). Multiscale testing of qualitative hypotheses. Annals of Statistics, 124-152.

[13] Eicker, F. (1979). The asymptotic distribution of the suprema of the standardized empirical processes. The Annals of Statistics, 116-138.

[14] Erdös, P. and A. Rényi (1970). On a new law of large numbers. Journal dAnalyse Mathématique 23(1), 103-111.

[15] Glaz, J. and N. Balakrishnan (Eds.) (2012). Scan Statistics and Applications. Springer Science \& Business Media.

[16] Glaz, J. and M. V. Koutras (Eds.) (2018+). Handbook of Scan Statistics. Springer, New York.

[17] Glaz, J., J. I. Naus, and S. Wallenstein (2001). Scan statistics. Springer.

[18] Glaz, J., V. Pozdnyakov, and S. Wallenstein (Eds.) (2009). Scan statistics: methods and applications. Springer Science \& Business Media.

[19] Gontscharuk, V. and H. Finner (2017). Asymptotics of goodness-of-fit tests based on minimum p-value statistics. Communications in Statistics-Theory and Methods 46(5), 2332-2342.

[20] Heffernan, R., F. Mostashari, D. Das, A. Karpati, M. Kulldorff, and D. Weiss (2004). Syndromic surveillance in public health practice, New York City. Emerging Infectious Diseases 10(5), 858-864.

[21] Jaeschke, D. (1979). The asymptotic distribution of the supremum of the standardized empirical distribution function on subintervals. The Annals of Statistics, 108-115.

[22] Jager, L. and J. A. Wellner (2004). A new goodness of fit test: the reversed berk-jones statistic.

[23] Jager, L. and J. A. Wellner (2007). Goodness-of-fit tests via phi-divergences. The Annals of Statistics 35(5), 2018-2053.

[24] Kabluchko, Z. (2011). Extremes of the standardized gaussian noise. Stochastic Processes and their Applications 121(3), 515-533.

[25] Kabluchko, Z. and Y. Wang (2014). Limiting distribution for the maximal standardized increment of a random walk. Stochastic Processes and their Applications 124(9), 2824-2867.

[26] Kolmogorov, A. (1933). Sulla determinazione empirica di una legge di distribuzione. Giornale dell'Istituto Italiano degli Attuari 4, 89-91.

[27] König, C., A. Munk, and F. Werner (2018). Multidimensional multiscale scanning in exponential families: Limit theory and statistical consequences. arXiv preprint arXiv:1802.07995. 
[28] Kulldorff, M. (1997). A spatial scan statistic. Communications in Statistics-Theory and methods 26(6), 1481-1496.

[29] Mack, C. (1948). An exact formula for $q_{k}(n)$, the probable number of $k$-aggregates in a random distribution of $n$ points. The London, Edinburgh, and Dublin Philosophical Magazine and Journal of Science 39(297), 778-790.

[30] Mason, D. M., G. R. Shorack, and J. A. Wellner (1983). Strong limit theorems for oscillation moduli of the uniform empirical process. Zeitschrift für Wahrscheinlichkeitstheorie und verwandte Gebiete 65(1), 83-97.

[31] Mikosch, T. and A. Račkauskas (2010). The limit distribution of the maximum increment of a random walk with regularly varying jump size distribution. Bernoulli 16(4), 1016-1038.

[32] Moscovich, A., B. Nadler, and C. Spiegelman (2016). On the exact berk-jones statistics and their $p$-value calculation. Electronic Journal of Statistics 10(2), 2329-2354.

[33] Naus, J. I. (1965). The distribution of the size of the maximum cluster of points on a line. Journal of the American Statistical Association 60(310), 532-538.

[34] Petrov, V. V. (1995). Limit theorems of probability theory: sequences of independent random variables. Technical report, Oxford, New York.

[35] Proksch, K., F. Werner, and A. Munk (2018). Multiscale scanning in inverse problems. The Annals of Statistics 46(6B), 3569-3602.

[36] Qualls, C. and H. Watanabe (1973). Asymptotic properties of gaussian random fields. Transactions of the American Mathematical Society 177, 155-171.

[37] Sharpnack, J. and E. Arias-Castro (2016). Exact asymptotics for the scan statistic and fast alternatives. Electronic Journal of Statistics 10(2), 2641-2684.

[38] Shorack, G. R. and J. A. Wellner (2009). Empirical processes with applications to statistics. SIAM.

[39] Siegmund, D. and E. Venkatraman (1995). Using the generalized likelihood ratio statistic for sequential detection of a change-point. The Annals of Statistics, 255-271.

[40] Silberstein, L. (1945). The probable number of aggregates in distributions of points. The London, Edinburgh, and Dublin Philosophical Magazine and Journal of Science 36(256), 319336. 